\title{
MOVEMENT OF CENTERS WITH RESPECT TO VARIOUS POTENTIALS
}

\author{
SHIGEHIRO SAKATA
}

ABstract. We investigate a potential with a radially symmetric and strictly decreasing kernel depending on a parameter. We regard the potential as a function defined on the upper half-space $\mathbb{R}^{m} \times(0,+\infty)$ and study some geometric properties of its spatial maximizer. To be precise, we give some sufficient conditions for the uniqueness of a maximizer of the potential and study the asymptotic behavior of the set of maximizers.

Using these results, we imply geometric properties of some specific potentials. In particular, we consider applications for the solution of the Cauchy problem for the heat equation, the Poisson integral (including a solid angle) and $r^{\alpha-m}$-potentials.

\section{INTRODUCTION}

Let $f: \mathbb{R}^{m} \rightarrow \mathbb{R}$ be a bounded measurable function with compact support. We consider a potential of the form

$$
K f(x, t)=\int_{\mathbb{R}^{m}} f(y) k(|x-y|, t) d y, x \in \mathbb{R}^{m}, t>0 .
$$

When $k(r, t)$ is given by the Gauss kernel $(4 \pi t)^{-m / 2} \exp \left(-r^{2} /(4 t)\right), K f(x, t)$ is the solution of the Cauchy problem for the heat equation with initial datum $f$, and its spatial maximum point for each $t$ is called a hot spot at time $t$. When $k(r, t)$ is given by the Poisson kernel $2 t\left(r^{2}+t^{2}\right)^{-(m+1) / 2} / \operatorname{Area}\left(S^{m}\right), K f(x, t)$ is the Poisson integral which is a solution of the Laplace equation for the upper half-space $\mathbb{R}^{m} \times(0,+\infty)$ with boundary datum $f$. When $k(r, \alpha)$ is given by $\operatorname{sign}(m-\alpha) r^{\alpha-m}$ if $0<\alpha \neq m$ or by $-\log r$ if $\alpha=m, K f(x, \alpha)$ is the $r^{\alpha-m}$-potential (an extension of the Newton potential or the Riesz potential), and its maximizer is called an $r^{\alpha-m}$-center, after O'Hara [11.

We remark that the kernel $k(r, t)$ is allowed to diverge at $r=0$ if the potential $K f(x, t)$ can be defined for any $x \in \mathbb{R}^{m}$. We suggest such a condition of $k(r, t)$ and show some basic properties of $K f$ with modification of the argument in [7, pp. 303318]. In particular, we give some differentiation formulas.

When $f$ is non-zero and non-negative, and when $k(r, t)$ is strictly decreasing with respect to $r$ for each $t$, the object of our investigation is a maximizer of $K f(\cdot, t): \mathbb{R}^{m} \rightarrow \mathbb{R}$. We call a maximizer of $K f(\cdot, t)$ a $k$-center of $f$ at time $t$. The existence of a $k$-center is rather straightforward (the idea of its proof is due

Received by the editors May 21, 2012 and, in revised form, March 14, 2013.

2010 Mathematics Subject Classification. Primary 31C12, 35K05, 35J05; Secondary 35B38, 51M16, 51M25, 52A40.

Key words and phrases. Hot spot, Poisson integral, illuminating center, solid angle, $r^{\alpha-m_{-}}$ potential, radial center, Newton potential, Riesz potential, Alexandrov's reflection principle, moving plane method, minimal unfolded region, heart. 
to $2,4,11$ ), but the uniqueness does not always hold. In fact, we can easily make an example where (at least) two $k$-centers exist in the case of the heat equation. Our first main result is a sufficient condition for the uniqueness; namely, if $k(r, t)$ is concave as a function of $r$, then $f$ has a unique $k$-center. In other words, the concavity of the kernel $k(\cdot, t)$ implies that of the potential $K f(\cdot, t)$. For example, if $k(r, \alpha)=-r^{\alpha-m}$ and $\alpha \geq m+1$, then $f$ has a unique $k$-center.

We next study the asymptotic behavior of the set of $k$-centers at time $t$ as $t$ goes to $+\infty$. It is well-known that any hot spot of the initial datum $f$ converges to the centroid (the center of mass) of $f$ as $t$ goes to $+\infty$, which was shown by Chavel and Karp in 4. Our second main result is to generalize the result in 4 ] in terms of the potential $K f(x, t)$; that is, we give a sufficient condition such that any $k$-center converges to the centroid of $f$ as $t$ goes to $+\infty$.

We also consider the case where $f$ coincides with the characteristic function of a body (the closure of a bounded open set) $\Omega$ in $\mathbb{R}^{m}$. We denote the potential $K \chi_{\Omega}$ by $K_{\Omega}$; that is,

$$
K_{\Omega}(x, t)=\int_{\Omega} k(|x-y|, t) d y, x \in \mathbb{R}^{m}, t>0 .
$$

We show some new differentiation formulas of the potential in this case. In particular, we show some boundary integral expressions of the derivative of $K_{\Omega}$ by using Stokes' theorem. Those expressions imply the superharmonicity of the potential $K_{\Omega}(\cdot, t)$ if $\Omega$ is convex.

When $k(r, t)$ is strictly decreasing as a function of $r$, we also investigate the properties of a maximizer of $K_{\Omega}(\cdot, t)$. In this case, we first give a new sufficient condition for the uniqueness of a $k$-center from geometric information about $\Omega$. To be precise, for a positive kernel $k$, if $\Omega$ is convex, and if $k(r, t) r^{m-1}$ is decreasing as a function of $r$, then $\Omega$ has a unique $k$-center. For example, if $k(r, \alpha)=r^{\alpha-m}$ and $0<\alpha \leq 1$, then any convex body $\Omega$ has a unique $k$-center.

We next restrict the location of a $k$-center. Using radial symmetry of the kernel and the philosophy of Alexandrov's reflection principle or the moving plane method ([5, 13]), we can construct a smaller region, depending only on $\Omega$, so that its complement has no chance of having any center in it. This region was introduced in 11 and called the minimal unfolded region of $\Omega$. When $\Omega$ is convex, the minimal unfolded region is also called the heart of $\Omega$, and it was independently defined by Brasco, Magnanini and Salani in [3] (see also [2]). But the idea of using the moving plane argument to restrict the region containing all $k$-centers dates back to [8].

It is easily shown that the center of a closed $m$-ball $B$ coincides with the unique $k$-center of $B$ by the moving plane method (or the minimal unfolded region of $B$ ). Using this fact, by the same argument as in [12, we show that if $B$ is a closed $m$-ball in $\mathbb{R}^{m}$ with the same volume as $\Omega$, and if $K_{B}$ is the potential defined in (1.2) for the same kernel as $K_{\Omega}$, then the maximum value of $K_{\Omega}$ is not greater than that of $K_{B}$. Moreover, equality holds if and only if $\Omega$ is a closed $m$-ball.

These investigations can be considered on the unit sphere $S^{m}$ or on the hyperbolic space $\mathbb{H}^{m}$. On these non-Euclidean spaces, we obtain the same results as in the case of the Euclidean space arguing in a similar way.

Studying geometric properties of maximizers of $K f(\cdot, t)$ originated in [11] by O'Hara. The consideration in [11] corresponds to the case of $r^{\alpha-m}$-potentials. He investigated the Hadamard finite part (the renormalization) of the $r^{\alpha-m}$-potential of the characteristic function $\chi_{\Omega}$ for $\alpha \leq 0$ and showed the uniqueness of a maximum 
point of the $r^{\alpha-m}$-potential when $\Omega$ is convex and $\alpha \leq 1$. On the other hand, it is easy to show that, for any $\Omega$, there is a unique maximum point of the $r^{\alpha-m_{-}}$ potential if $\alpha \geq m+1$. Thus he obtained a generalization of the centroid of a body $\Omega$ which corresponds to the case of $\alpha=m+2$. In this connection, if $\Omega$ is convex, then an $r^{\alpha-m}$-center is also called a radial center of order $\alpha$ introduced by Moszyńska in [10.

This type of research also originated in 14 by Shibata. The consideration in [14] corresponds to the case of the Poisson integral. He introduced a spatial illuminating center of a triangle $\triangle$ in $\mathbb{R}^{2}$ of height $h>0$. The center of $\triangle$ is defined as a point that maximizes the "total brightness" of a triangular park $\triangle$ when a light source is located above that point by height $h$. It is easy to see that the total brightness is proportional to the Poisson integral; namely, the total brightness is given by

$$
\mathbb{R}^{2} \ni x \mapsto \int_{\triangle} \frac{h}{\left(|x-y|^{2}+h^{2}\right)^{3 / 2}} d y \in \mathbb{R} .
$$

This function also coincides with the solid angle of $\triangle$ at the light source. Let us generalize Shibata's notion for a body $\Omega$ in $\mathbb{R}^{m}$. Then we imply some basic results on the generalized total brightness of $\Omega$ and spatial illuminating centers as byproducts of our discussion on $K_{\Omega}$. Moreover, by using the explicit form of the kernel, we show a new sufficient condition for the uniqueness of a spatial illuminating center.

The consideration about $k$-centers also includes that about hot spots as a specific example. Therefore, it is expected that we obtain some properties of $K f(x, t)$ in the same manner as in [4. Let us generalize the notion in [4 for our potential. After the generalization, we show that the set of maximum points of the Poisson integral has similar properties to that of hot spots.

Furthermore, we show a new property about hot spots. It is known that if $t \geq(\operatorname{diam}(\operatorname{supp} f))^{2} / 2$, then $f$ has a unique hot spot at time $t$, which was indicated by Jimbo and Sakaguchi in [6]. In particular, we consider the case where the initial datum $f$ coincides with the characteristic function of a body $\Omega$. Then we can slightly improve the condition of $t$ given in [6].

Summarizing the above, our framework in this paper can be applied to such examples and presents a new viewpoint to study them.

Throughout this paper, $X^{c}, \bar{X}, \stackrel{\circ}{X}$, conv $X$ and diam $X$ denote the complement, the closure, the interior, the convex hull and the diameter of a set $X$ in $\mathbb{R}^{m}$, respectively. We denote the Lebesgue surface measure of any $N$-dimensional surface by $\sigma_{N}$. In particular, the symbol $\sigma$ is used in the case of $N=m-1$, for short.

\section{Preliminaries}

Throughout this paper, the conditions $(B),\left(C_{\alpha}^{0}\right),\left(C_{\alpha}^{1}\right)$ and $\left(C_{\alpha}^{2}\right)$ for a function $\varphi:(0,+\infty) \rightarrow \mathbb{R}$ are defined as follows:

$(B) \varphi$ can be defined at 0 .

$\left(C_{\alpha}^{0}\right) \alpha>0, \varphi$ is continuous on the interval $(0,+\infty)$, and

$$
\varphi(r)= \begin{cases}O\left(r^{\alpha-m}\right) & (\alpha<m), \\ O(\log r) & (\alpha=m), \\ O(1) & (\alpha>m),\end{cases}
$$

as $r \rightarrow 0^{+}$. 
$\left(C_{\alpha}^{1}\right) \alpha>1, \varphi$ is of class $C^{1}$ on the interval $(0,+\infty)$ and satisfies the condition $\left(C_{\alpha}^{0}\right)$, and

$$
\varphi^{\prime}(r)= \begin{cases}O\left(r^{\alpha-m-1}\right) & (\alpha<m+1), \\ O(\log r) & (\alpha=m+1), \\ O(1) & (\alpha>m+1),\end{cases}
$$

as $r \rightarrow 0^{+}$

$\left(C_{\alpha}^{2}\right) \alpha>2, \varphi$ is of class $C^{2}$ on the interval $(0,+\infty)$ and satisfies the condition $\left(C_{\alpha}^{1}\right)$, and

$$
\varphi^{\prime \prime}(r)= \begin{cases}O\left(r^{\alpha-m-2}\right) & (\alpha<m+2), \\ O(\log r) & (\alpha=m+2) \\ O(1) & (\alpha>m+2)\end{cases}
$$

as $r \rightarrow 0^{+}$.

Let $f: \mathbb{R}^{m} \rightarrow \mathbb{R}$ be a bounded measurable function with compact support. We consider a kernel $k:(0,+\infty) \times(0,+\infty) \rightarrow \mathbb{R}$ such that $k(\cdot, t):(0,+\infty) \rightarrow \mathbb{R}$ satisfies any of the conditions $(B),\left(C_{\alpha}^{0}\right),\left(C_{\alpha}^{1}\right)$ or $\left(C_{\alpha}^{2}\right)$ for each $t>0$. Let

$$
K f(x, t)=\int_{\mathbb{R}^{m}} f(y) k(r, t) d y, x \in \mathbb{R}^{m}, t>0, r=|x-y| .
$$

We remark that if $k(\cdot, t)$ satisfies the condition $(B)$, then $K f(x, t)$ is defined for any $x \in \mathbb{R}^{m}$. But, for other cases, the well-definedness of $K f(x, t)$ is non-trivial. (It will be shown later.)

Under the condition $\left(C_{\alpha}^{i}\right)$, let

$$
\tilde{C}_{\alpha}^{i}(t)= \begin{cases}\lim _{r \rightarrow 0^{+}}\left|\frac{\partial^{i} k}{\partial r^{i}}(r, t) / r^{\alpha-m-i}\right| & (\alpha<m+i), \\ \lim _{r \rightarrow 0^{+}}\left|\frac{\partial^{i} k}{\partial r^{i}}(r, t) / \log r\right| & (\alpha=m+i), \\ \lim _{r \rightarrow 0^{+}}\left|\frac{\partial^{i} k}{\partial r^{i}}(r, t)\right| & (\alpha>m+i),\end{cases}
$$

and let

$$
C_{\alpha}^{i}(t)=\max \left\{2 \max _{0 \leq j \leq i} \tilde{C}_{\alpha}^{j}(t), 1\right\}
$$

in what follows. Here, we used the notation $\partial^{0} k / \partial r^{0}=k$.

We denote a point in $\mathbb{R}^{m}$ by $x=\left(x_{1}, \ldots, x_{m}\right)$ and a point in $\operatorname{supp} f$ by $y=$ $\left(y_{1}, \ldots, y_{m}\right)$. We understand that the letter $r$ is always used for $r=|x-y|$. We denote the closed $m$-ball centered at $x$ with radius $\varepsilon>0$ by $B_{\varepsilon}(x)$.

2.1. Basic properties of $K f$ under the condition $\left(C_{\alpha}^{0}\right)$. In this subsection, we assume that $k(\cdot, t)$ satisfies the condition $\left(C_{\alpha}^{0}\right)$ for a positive $t$.

Lemma 2.1. Let $0<\alpha<m$. There exists an $\varepsilon^{\prime}>0$ such that if $0<\varepsilon<\varepsilon^{\prime}$, then

$$
\int_{B_{\varepsilon}(x)}|k(r, t)| d y \leq \frac{C_{\alpha}^{0}(t) \sigma\left(S^{m-1}\right) \varepsilon^{\alpha}}{\alpha}
$$

for any $x \in \mathbb{R}^{m}$. 
Proof. There exists an $\varepsilon^{\prime}>0$ such that, for any $0<r<\varepsilon^{\prime},|k(r, t)| \leq C_{\alpha}^{0}(t) r^{\alpha-m}$; and fix such an $\varepsilon^{\prime}$. Hence we obtain

$$
\int_{B_{\varepsilon}(x)}|k(r, t)| d y \leq C_{\alpha}^{0}(t) \int_{B_{\varepsilon}(x)} r^{\alpha-m} d y=\frac{C_{\alpha}^{0}(t) \sigma\left(S^{m-1}\right) \varepsilon^{\alpha}}{\alpha}
$$

if $0<\varepsilon<\varepsilon^{\prime}$.

Corollary 2.2. For any $\alpha>0$, we have

$$
\lim _{\varepsilon \rightarrow 0} \int_{B_{\varepsilon}(x)}|k(r, t)| d y=0
$$

uniformly on $\mathbb{R}^{m}$.

Proposition 2.3. $K f(\cdot, t)$ is defined and continuous on $\mathbb{R}^{m}$.

Proof. Fix a small enough positive constant $\varepsilon$. We remark that we have

$$
|K f(x, t)| \leq\|f\|_{\infty}\left(\int_{B_{\varepsilon}(x)}|k(r, t)| d y+\int_{\operatorname{supp} f \backslash B_{\varepsilon}(x)}|k(r, t)| d y\right) .
$$

By Lemma 2.1, the first integral is bounded on $\mathbb{R}^{m}$. Since $k(r, t)$ is continuous on the compact set supp $f \backslash \stackrel{\circ}{B}_{\varepsilon}(x)$ as a function of $y$ for each $x$, the second integral is also bounded. Hence $K f(\cdot, t)$ is defined on $\mathbb{R}^{m}$.

It is sufficient to show that $K f(\cdot, t)$ is continuous on an arbitrary bounded open set $F$ containing supp $f$. In order to show the continuity of $K f(\cdot, t)$ on $F$, let

$$
K_{\varepsilon} f(x, t)=\int_{\mathbb{R}^{m}} f(y) k(r, t) \psi\left(\frac{r}{\varepsilon}\right) d y, x \in \mathbb{R}^{m},
$$

where $\psi$ is a $C^{2}$ function which satisfies the following conditions:

$$
\psi(r)=0 \text { if } 0 \leq r \leq 1,0 \leq \psi^{\prime}(r) \leq 2 \text { if } 1 \leq r \leq 2 \text {, and } \psi(r)=1 \text { if } 2 \leq r .
$$

Since we have

$$
\left|k(r, t) \psi\left(\frac{r}{\varepsilon}\right)\right| \leq \sup _{\varepsilon \leq r \leq \operatorname{diam} F}|k(r, t)|<+\infty
$$

for any $x \in F$ and $y \in \operatorname{supp} f, K_{\varepsilon} f(\cdot, t)$ is defined on $F$. For an arbitrary point $x \in F$ and an arbitrary sequence $\{x(\ell)\} \subset F$ converging to $x$, we have $K_{\varepsilon} f(x(\ell), t)$ converges to $K_{\varepsilon} f(x, t)$ as $\ell$ goes to $+\infty$ by Lebesgue's dominated convergence theorem. Hence $K_{\varepsilon} f(\cdot, t)$ is continuous on $F$.

Then we have

$$
\begin{aligned}
\left|\left(K-K_{\varepsilon}\right) f(x, t)\right| & \leq\|f\|_{\infty} \int_{\operatorname{supp} f}\left|k(r, t)\left(1-\psi\left(\frac{r}{\varepsilon}\right)\right)\right| d y \\
& \leq\|f\|_{\infty} \int_{B_{2 \varepsilon}(x)}|k(r, t)| d y,
\end{aligned}
$$

and Corollary 2.2 implies that $K f(\cdot, t)$ converges to $K_{\varepsilon} f(\cdot, t)$ as $\varepsilon$ tends to zero uniformly on $F$. Hence $K f(\cdot, t)$ is continuous on $F$. 
2.2. Basic properties of $K f$ under the condition $\left(C_{\alpha}^{1}\right)$. In this subsection, we assume that $k(\cdot, t)$ satisfies the condition $\left(C_{\alpha}^{1}\right)$ for a positive $t$.

Lemma 2.4. Let $1<\alpha<m+1$. There exists an $\varepsilon^{\prime}>0$ such that if $0<\varepsilon<\varepsilon^{\prime}$, then

$$
\int_{B_{\varepsilon}(x)}\left|\frac{\partial}{\partial x_{j}} k(r, t)\right| d y \leq \frac{C_{\alpha}^{1}(t) \sigma\left(S^{m-1}\right) \varepsilon^{\alpha-1}}{\alpha-1}
$$

for any $x \in \mathbb{R}^{m}$.

Proof. We can show this statement in the same manner as in Lemma 2.1

Corollary 2.5. For any $\alpha>1$, we have

$$
\lim _{\varepsilon \rightarrow 0} \int_{B_{\varepsilon}(x)}\left|\frac{\partial}{\partial x_{j}} k(r, t)\right| d y=0
$$

uniformly on $\mathbb{R}^{m}$.

Proposition 2.6. $K f(\cdot, t)$ is of class $C^{1}$ on $\mathbb{R}^{m}$, and we have

$$
\frac{\partial K f}{\partial x_{j}}(x, t)=\int_{\mathbb{R}^{m}} f(y) \frac{\partial}{\partial x_{j}} k(r, t) d y
$$

for any $x \in \mathbb{R}^{m}$.

Proof. It is sufficient to show that the statement holds on an arbitrary bounded open set $F$ containing $\operatorname{supp} f$.

Let

$$
g(x, t)=\int_{\mathbb{R}^{m}} f(y) \frac{\partial}{\partial x_{j}} k(r, t) d y
$$

for $x \in F$. We can show the well-definedness (convergence) and the continuity of $g(\cdot, t)$ on $F$ in the same manner as in those of $K f(\cdot, t)$ (see Proposition 2.3) by using the function

$$
g_{\varepsilon}(x, t)=\int_{\mathbb{R}^{m}}\left(f(y) \frac{\partial}{\partial x_{j}} k(r, t)\right) \psi\left(\frac{r}{\varepsilon}\right) d y, x \in \mathbb{R}^{m},
$$

where $\psi$ is defined in (2.8).

It suffices to show that the first derivative of $K_{\varepsilon} f(\cdot, t)$ is defined and converges to $g(\cdot, t)$ uniformly on $F$, where $K_{\varepsilon} f$ is defined in (2.7). For any $x \in F$ and $y \in \operatorname{supp} f$, we have

$$
\left|\frac{\partial}{\partial x_{j}}\left(k(r, t) \psi\left(\frac{r}{\varepsilon}\right)\right)\right| \leq \sup _{\varepsilon \leq r \leq \operatorname{diam} F}\left|\frac{\partial k}{\partial r}(r, t)\right|+\frac{2}{\varepsilon} \sup _{\varepsilon \leq r \leq \operatorname{diam} F}|k(r, t)|<+\infty .
$$

Hence the first derivative $\left(\partial K_{\varepsilon} f / \partial x_{j}\right)(x, t)$ exists as

$$
\begin{aligned}
& \lim _{\lambda \rightarrow 0} \frac{K_{\varepsilon} f\left(x+\lambda e_{j}, t\right)-K_{\varepsilon} f(x, t)}{\lambda} \\
= & \int_{\mathbb{R}^{m}} f(y) \lim _{\lambda \rightarrow 0} \frac{k\left(\left|x+\lambda e_{j}-y\right|, t\right) \psi\left(\frac{\left|x+\lambda e_{j}-y\right|}{\varepsilon}\right)-k(|x-y|, t) \psi\left(\frac{|x-y|}{\varepsilon}\right)}{\lambda} d y \\
= & \int_{\mathbb{R}^{m}} f(y) \frac{\partial}{\partial x_{j}}\left(k(r, t) \psi\left(\frac{r}{\varepsilon}\right)\right) d y \in \mathbb{R}
\end{aligned}
$$


by Lebesgue's dominated convergence theorem, where $e_{j}$ is the $j$-th unit vector of $\mathbb{R}^{m}$. Then we get that $\left(\partial K_{\varepsilon} f / \partial x_{j}\right)(x, t)$ converges to $g(x, t)$ as $\varepsilon$ tends to zero uniformly on $F$ by Lemma 2.1 and Corollary 2.5 in the same manner as in Proposition 2.3 .

2.3. Basic properties of $K f$ under the condition $\left(C_{\alpha}^{2}\right)$. In this subsection, we assume that $k(\cdot, t)$ satisfies the condition $\left(C_{\alpha}^{2}\right)$ for a positive $t$.

Lemma 2.7. Let $2<\alpha<m+1$. There exists an $\varepsilon^{\prime}>0$ such that if $0<\varepsilon<\varepsilon^{\prime}$, then

$$
\int_{B_{\varepsilon}(x)}\left|\frac{\partial^{2}}{\partial x_{i} \partial x_{j}} k(r, t)\right| d y \leq \frac{2 C_{\alpha}^{2}(t) \sigma\left(S^{m-1}\right) \varepsilon^{\alpha-2}}{\alpha-2}
$$

for any $x \in \mathbb{R}^{m}$.

Proof. There exists an $\varepsilon^{\prime}>0$ such that, for any $0<r<\varepsilon^{\prime}$, the following inequalities hold:

$$
\left|\frac{\partial^{2} k}{\partial r^{2}}(r, t)\right| \leq C_{\alpha}^{2}(t) r^{\alpha-m-2},\left|\frac{\partial k}{\partial r}(r, t)\right| \leq C_{\alpha}^{2}(t) r^{\alpha-m-1} .
$$

The rest is similar to the proof of Lemma 2.1.

Corollary 2.8. For any $\alpha>2$, we have

$$
\lim _{\varepsilon \rightarrow 0} \int_{B_{\varepsilon}(x)}\left|\frac{\partial^{2}}{\partial x_{i} \partial x_{j}} k(r, t)\right| d y=0
$$

uniformly on $\mathbb{R}^{m}$.

Proposition 2.9. $K f(\cdot, t)$ is of class $C^{2}$ on $\mathbb{R}^{m}$, and we have

$$
\frac{\partial^{2} K f}{\partial x_{i} \partial x_{j}}(x, t)=\int_{\mathbb{R}^{m}} f(y) \frac{\partial^{2}}{\partial x_{i} \partial x_{j}} k(r, t) d y,
$$

for any $x \in \mathbb{R}^{m}$.

Proof. It is sufficient to show that the statement holds on an arbitrary bounded open set $F$ containing supp $f$.

Let

$$
g(x, t)=\int_{\mathbb{R}^{m}} f(y) \frac{\partial^{2}}{\partial x_{i} \partial x_{j}} k(r, t) d y
$$

for $x \in F$. We can show the well-definedness (convergence) and the continuity of $g(\cdot, t)$ on $F$ in the same manner as in those of $K f(\cdot, t)$ (see Proposition 2.3) by using the function

$$
g_{\varepsilon}(x, t)=\int_{\mathbb{R}^{m}}\left(f(y) \frac{\partial^{2}}{\partial x_{i} \partial x_{j}} k(r, t)\right) \psi\left(\frac{r}{\varepsilon}\right) d y, x \in \mathbb{R}^{m},
$$

where $\psi$ is defined in (2.8).

The rest is similar to the calculation as in Proposition 2.6 that is, we can show that the second derivative of $K_{\varepsilon} f(\cdot, t)$ is defined and converges to $g(\cdot, t)$ as $\varepsilon$ tends to zero uniformly on $F$. 


\section{Results On $k$-CEnTERS}

3.1. Existence and uniqueness of a $k$-center, energy of $K f$. In this subsection, we assume that $f$ is non-zero and non-negative. We understand that $f$ is non-zero if the set of points that give non-zero values to $f$ has a positive measure. Moreover, we assume that $k(r, t)$ is strictly decreasing with respect to $r$ for each $t$. We remark that the results in this subsection can be applied to the case where $k(r, t)$ is strictly increasing with respect to $r$ by considering the kernel $-k(r, t)$.

The ideas of the proofs of Lemma 3.1] and Proposition 3.2 are due to [2] (see also [4, 11]).

Lemma 3.1. For any positive constant $t, K f(\cdot, t)$ is upper semi-continuous on $\mathbb{R}^{m}$.

Proof. It suffices to show the statement under the condition $(B)$. Thanks to Fatou's lemma, for any $x \in \mathbb{R}^{m}$, we have

$$
\limsup _{\xi \rightarrow x} K f(\xi, t) \leq \int_{\mathbb{R}^{m}} f(y) \limsup _{\xi \rightarrow x} k(|\xi-y|, t) d y=K f(x, t)
$$

Here, we use the fact that any monotonic function only has at most countable discontinuous points.

Proposition 3.2. For any positive constant $t$, there exists a maximum point of $K f(\cdot, t)$, and all the possible maximum points are contained in the convex hull of the support of $f$.

Proof. Since $K f(\cdot, t)$ is upper semi-continuous on $\mathbb{R}^{m}$ for any $t>0$, and since $\operatorname{conv}(\operatorname{supp} f)$ is compact, there exists a point $x$ that gives the maximum value of $K f(\cdot, t)$ in $\operatorname{conv}(\operatorname{supp} f)$ when we restrict the domain of $K f(\cdot, t)$ to $\operatorname{conv}(\operatorname{supp} f)$.

We show that the point $x$ gives the maximum value of $K f(\cdot, t)$. For any point $x^{\prime}$ in the complement of $\operatorname{conv}(\operatorname{supp} f)$, we can choose a point $x^{\prime \prime}$ on the boundary of $\operatorname{conv}(\operatorname{supp} f) \operatorname{such}$ that $\operatorname{dist}\left(x^{\prime}, \partial(\operatorname{conv}(\operatorname{supp} f))\right)$ is attained by the point. Then $\operatorname{conv}(\operatorname{supp} f)$ is contained in a half-space whose boundary is the hyperplane orthogonal to the line through $x^{\prime}$ and $x^{\prime \prime}$. Hence we have $\left|x^{\prime}-y\right|>\left|x^{\prime \prime}-y\right|$ for any point $y$ in $\operatorname{supp} f$, and hence $K f\left(x^{\prime}, t\right)<K f\left(x^{\prime \prime}, t\right) \leq K f(x, t)$.

Definition 3.3. A point $x$ is called a $k$-center of $f$ at time $t$ if it gives the maximum value of $K f(\cdot, t)$. We denote the set of $k$-centers of $f$ at time $t$ by $\mathcal{K}_{f}(t)$; that is,

$$
\mathcal{K}_{f}(t)=\left\{x \in \mathbb{R}^{m} \mid K f(x, t)=\max _{\xi \in \mathbb{R}^{m}} K f(\xi, t)\right\} .
$$

Theorem 3.4. Let $B_{f}$ be the minimum closed ball containing $\operatorname{conv}(\operatorname{supp} f)$. If $k(\cdot, t)$ is concave on the interval $(0, \operatorname{diam}(\operatorname{supp} f)]$, then the potential $K f(\cdot, t)$ is strictly concave on the ball $B_{f}$. 
Proof. Fix two points $x$ and $x^{\prime}$ in $B_{f}$ arbitrarily. By the strictly decreasing behavior and the concavity of $k(\cdot, t)$, we have

$$
\begin{aligned}
K f\left(\frac{x+x^{\prime}}{2}, t\right) & =\int_{\mathbb{R}^{m}} f(y) k\left(\left|\frac{x+x^{\prime}}{2}-y\right|, t\right) d y \\
& >\int_{\mathbb{R}^{m}} f(y) k\left(\frac{|x-y|+\left|x^{\prime}-y\right|}{2}, t\right) d y \\
& \geq \int_{\mathbb{R}^{m}} f(y) \frac{k(|x-y|, t)+k\left(\left|x^{\prime}-y\right|, t\right)}{2} d y \\
& =\frac{K f(x, t)+K f\left(x^{\prime}, t\right)}{2}
\end{aligned}
$$

which completes the proof.

Corollary 3.5. If $k(\cdot, t)$ is concave on the interval $(0, \operatorname{diam}(\operatorname{supp} f)]$, then $f$ has a unique $k$-center.

Morgan considered a variational problem for a potential energy in [9]. In order to apply his result to our case, we set

$$
\mathcal{F}=\left\{f \in L_{c}^{\infty}\left(\mathbb{R}^{m}\right) \mid 0 \leq f \leq 1, \int_{\mathbb{R}^{m}} f(y) d y=1\right\} .
$$

Theorem 3.6 (9]). Suppose that $k$ is a positive function. Then, for each $t$, the potential energy

$$
E_{t}(f)=\int_{\mathbb{R}^{m}} f(x) K f(x, t) d x=\int_{\mathbb{R}^{m} \times \mathbb{R}^{m}} f(x) f(y) k(r, t) d x d y
$$

is maximum in $\mathcal{F}$ if and only if $f$ is the characteristic function of a closed $m$-ball up to a zero measure set.

3.2. A separation of variables form. In this subsection, we assume that $f$ is non-zero and non-negative. Moreover, we assume that $k(r, t)$ is strictly decreasing with respect to $r$ for each $t$. We remark that the results in this subsection can be applied to the case where $k(r, t)$ is strictly increasing with respect to $r$ by considering the kernel $-k(r, t)$.

We study the asymptotic behavior of the set of maximum points $\mathcal{K}_{f}(t)$ as $t$ goes to $+\infty$ when the kernel $k(r, t)$ is given by the separation of variables form

$$
k(r, t)=\bar{k}\left(\varphi(r) \psi_{1}(t)+\psi_{2}(t)\right) .
$$

We understand that the kernel $k(r, t)$ is expressed as the form (3.2) on the rectangle $(0, R) \times(T,+\infty)$ if the function $\bar{k}$ is defined on the interval

$$
\mathcal{I}\left(\varphi, \psi_{1}, \psi_{2} ; R, T\right)=\overline{\left(\inf \left(\varphi(r) \psi_{1}(t)+\psi_{2}(t)\right), \sup \left(\varphi(r) \psi_{1}(t)+\psi_{2}(t)\right)\right)} .
$$

Here, we take the infimum and the supremum over the rectangle $(0, R) \times(T,+\infty)$, and the over-bar means the closure in $\mathbb{R}$ with respect to the Euclidean norm.

For any strictly monotonic function $\varphi:(0,+\infty) \rightarrow \mathbb{R}$ which satisfies the condition $\left(C_{\beta}^{1}\right)$, we denote the set of critical points of the following potential by $\mathcal{C}(\varphi, f)$ :

$$
\mathbb{R}^{m} \ni x \mapsto \int_{\mathbb{R}^{m}} f(y) \varphi(r) d y \in \mathbb{R} .
$$

Thanks to Proposition [3.2, $\mathcal{C}(\varphi, f)$ is not empty. We denote the the $\varepsilon$-tubular neighborhood of $\mathcal{C}(\varphi, f)$ by $N_{\varepsilon}(\mathcal{C}(\varphi, f))$. 
Lemma 3.7. Suppose that there exists a non-negative constant $T$ such that if $t>T$, then $k(\cdot, t)$ satisfies the condition $\left(C_{\alpha}^{1}\right)$. Moreover, we assume that there exist functions $\varphi, \psi_{1}, \psi_{2}$ and $\bar{k}$ which satisfy the following conditions:

- $k(r, t)$ can be expressed as $k(r, t)=\bar{k}\left(\varphi(r) \psi_{1}(t)+\psi_{2}(t)\right)$ on the rectangle $(0, \operatorname{diam}(\operatorname{supp} f)] \times(T,+\infty)$.

- $\varphi$ is a strictly monotonic function satisfying the condition $\left(C_{\beta}^{1}\right)$ for some $\beta>(m+1) / 2$.

- $\psi_{1}(t)$ tends to 0 , and $\psi_{2}(t)$ converges to a constant $a \in \mathbb{R}$ as $t$ goes to $+\infty$.

- $\bar{k}$ is of class $C^{2}$, the first derivative $\bar{k}^{\prime}$ does not vanish at a, and the second derivative $\bar{k}^{\prime \prime}$ is bounded.

For any positive $\varepsilon$, there exists a constant $T^{\prime} \geq T$ such that if $t \geq T^{\prime}$, and if $x \in(\operatorname{conv}(\operatorname{supp} f)) \backslash N_{\varepsilon}(\mathcal{C}(\varphi, f))$, then the first derivative of $K f(\cdot, t)$ does not vanish for some directions.

Proof. We remark that, for any $x \in \operatorname{conv}(\operatorname{supp} f)$,

$$
\begin{aligned}
K f(x, t)= & \bar{k}^{\prime}(a) \psi_{1}(t) \int_{\mathbb{R}^{m}} f(y) \varphi(r) d y \\
& +\int_{\mathbb{R}^{m}} f(y)\left(\bar{k}\left(\varphi(r) \psi_{1}(t)+\psi_{2}(t)\right)-\bar{k}^{\prime}(a) \varphi(r) \psi_{1}(t)\right) d y
\end{aligned}
$$

Fix an arbitrary $\varepsilon>0$, and let

$$
L(\varepsilon)=\left|\bar{k}^{\prime}(a)\right| \max _{1 \leq j \leq m}\left(\inf _{x \in(\operatorname{conv}(\operatorname{supp} f)) \backslash N_{\varepsilon}(\mathcal{C}(\varphi, f))}\left|\frac{\partial}{\partial x_{j}} \int_{\mathbb{R}^{m}} f(y) \varphi(r) d y\right|\right)>0 .
$$

Let $\delta=\left(\left|\psi_{1}(t)\right|^{2} / C_{\alpha}^{1}(t)\right)^{1 /(\alpha-1)}$, where $C_{\alpha}^{1}(t)$ is defined in equation (2.6). Thanks to Lemma 2.1. Lemma 2.4 and the mean value theorem, there exist positive constants $M_{1}, M_{2}, M_{3}$ and $M_{4}$ such that, for any large enough positive $t$ and $x \in$ $\operatorname{conv}(\operatorname{supp} f)$, we have

$$
\begin{aligned}
& \left|\frac{\partial}{\partial x_{j}} \int_{\mathbb{R}^{m}} f(y)\left(\bar{k}\left(\varphi(r) \psi_{1}(t)+\psi_{2}(t)\right)-\bar{k}^{\prime}(a) \varphi(r) \psi_{1}(t)\right) d y\right| \\
\leq & \int_{\mathbb{R}^{m} \backslash B_{\delta}(x)} f(y)\left|\frac{\partial}{\partial x_{j}}\left(\bar{k}\left(\varphi(r) \psi_{1}(t)+\psi_{2}(t)\right)-\bar{k}^{\prime}(a) \varphi(r) \psi_{1}(t)\right)\right| d y \\
& +\int_{B_{\delta}(x)} f(y)\left|\frac{\partial}{\partial x_{j}} \bar{k}\left(\varphi(r) \psi_{1}(t)+\psi_{2}(t)\right)\right| d y \\
& +\left|\bar{k}^{\prime}(a) \psi_{1}(t)\right| \int_{B_{\delta}(x)} f(y)\left|\frac{\partial}{\partial x_{j}} \varphi(r)\right| d y \\
\leq & \left|\psi_{1}(t)\right| \int_{\mathbb{R}^{m} \backslash B_{\delta}(x)} f(y)\left|\varphi^{\prime}(r)\right|\left|\bar{k}^{\prime}\left(\varphi(r) \psi_{1}(t)+\psi_{2}(t)\right)-\bar{k}^{\prime}(a)\right| d y+M_{3} C_{\alpha}^{1}(t) \delta^{\alpha-1} \\
& +M_{4}\left|\psi_{1}(t)\right| \delta^{\beta-1} \\
\leq & \left\|\bar{k}^{\prime \prime}\right\|_{\infty}\left|\psi_{1}(t)\right| \int_{\mathbb{R}^{m}} f(y)\left|\varphi^{\prime}(r)\right|\left|\varphi(r) \psi_{1}(t)+\psi_{2}(t)-a\right| d y+M_{3} C_{\alpha}^{1}(t) \delta^{\alpha-1} \\
& +M_{4}\left|\psi_{1}(t)\right| \delta^{\beta-1}
\end{aligned}
$$




$$
\begin{aligned}
\leq & \left\|\bar{k}^{\prime \prime}\right\|_{\infty}\left|\psi_{1}(t)\right|\left(\left|\psi_{1}(t)\right| \int_{\mathbb{R}^{m}} f(y)\left|\varphi^{\prime}(r) \varphi(r)\right| d y+\left|\psi_{2}(t)-a\right| \int_{\mathbb{R}^{m}} f(y)\left|\varphi^{\prime}(r)\right| d y\right) \\
& +M_{3} C_{\alpha}^{1}(t) \delta^{\alpha-1}+M_{4}\left|\psi_{1}(t)\right| \delta^{\beta-1} \\
\leq & \left|\psi_{1}(t)\right|\left(M_{1}\left|\psi_{1}(t)\right|+M_{2}\left|\psi_{2}(t)-a\right|+M_{3}\left|\psi_{1}(t)\right|+M_{4}\left|\psi_{1}(t)\right|^{2(\beta-1) /(\alpha-1)}\right) .
\end{aligned}
$$

By the assumptions of $\psi_{1}$ and $\psi_{2}$, for any large enough positive $t$, we obtain

$$
\begin{aligned}
& \max _{1 \leq j \leq m}\left(\inf _{x \in(\operatorname{conv}(\operatorname{supp} f)) \backslash N_{\varepsilon}(\mathcal{C}(\varphi, f))}\left|\frac{\partial K f}{\partial x_{j}}(x, t)\right|\right) /\left|\psi_{1}(t)\right| \\
\geq & L(\varepsilon)-\left(M_{1}\left|\psi_{1}(t)\right|+M_{2}\left|\psi_{2}(t)-a\right|+M_{3}\left|\psi_{1}(t)\right|+M_{4}\left|\psi_{1}(t)\right|^{2(\beta-1) /(\alpha-1)}\right) \\
> & 0,
\end{aligned}
$$

which completes the proof.

Theorem 3.8. Let $k$ be as in Lemma 3.7. For any strictly increasing sequence $\left\{t_{\ell}\right\}$ and any $k$-center $c\left(t_{\ell}\right) \in \mathcal{K}_{f}\left(t_{\ell}\right)$, if $\left\{t_{\ell}\right\}$ diverges to $+\infty$ as $\ell$ goes to $+\infty$, then the distance between $c\left(t_{\ell}\right)$ and $\mathcal{C}(\varphi, f)$ tends to 0 as $\ell$ goes to $+\infty$.

Corollary 3.9. Let $k$ be as in Lemma 3.7. If either $\varphi$ or $-\varphi$ is concave and strictly decreasing on the interval $(0, \operatorname{diam}(\operatorname{supp} f)]$, then the set of $k$-centers $\mathcal{K}_{f}(t)$ converges to the set $\mathcal{C}(\varphi, f) \cap \operatorname{conv}(\operatorname{supp} f)$ with respect to the Hausdorff distance as $t$ goes to $+\infty$.

Proof. Thanks to Theorem 3.4, the set $\mathcal{C}(\varphi, f) \cap \operatorname{conv}(\operatorname{supp} f)$ consists of one point. Hence Theorem 3.8 implies the conclusion.

Remark 3.10. Under the same assumptions as in Lemma 3.7, if the set $\mathcal{C}(\varphi, f) \cap$ $\operatorname{conv}(\operatorname{supp} f)$ becomes a one-point set, then we obtain the same conclusion as in Corollary 3.9 .

Remark 3.11. In section 5, we consider some applications of Corollary 3.5 and Corollary 3.9. Then we use the case where $\varphi(r)=r^{\beta-m}$ for some $\beta>m, \psi_{1}(t)=t^{p}$ for some positive $p$ and $\psi_{2}(t)=0$.

3.3. A special separation of variables form. In this subsection, we assume that $f$ has a non-zero mass; that is,

$$
\int_{\mathbb{R}^{m}} f(y) d y \neq 0 .
$$

We consider the case where $k(r, t)$ is given as

$$
k(r, t)=\bar{k}\left(r^{2} \psi_{1}(t)+\psi_{2}(t)\right) .
$$

The theorems and those proofs in this subsection are based on [4].

Remark 3.12. The centroid (the center of mass) of $f$, denoted by $G_{f}$, is given as

$$
G_{f}=\int_{\mathbb{R}^{m}} f(y) y d y / \int_{\mathbb{R}^{m}} f(y) d y .
$$

In [11, O'Hara indicated that the centroid of a body (the closure of a bounded open set) $\Omega$ in $\mathbb{R}^{m}$ can be obtained as the (unique) critical point of the potential $V_{\Omega}^{(m+2)}$ defined in (5.9). We can show that, by the same argument, the centroid of $f$ can be obtained as the (unique) critical point of the potential $V^{(m+2)} f$ defined in (5.10). Hence the set $\mathcal{C}\left(r^{2}, f\right)$ coincides with the one-point set $\left\{G_{f}\right\}$. 
We first investigate the asymptotic behavior of level sets of $K f(\cdot, t)$.

Theorem 3.13. Suppose that there exists a non-negative constant $T$ such that $k(\cdot, t)$ satisfies the condition $\left(C_{\alpha}^{1}\right)$ for any $t>T$. Moreover, we assume that there exist functions $\psi_{1}, \psi_{2}$ and $\bar{k}$ which satisfy the following conditions:

- $k(r, t)$ can be expressed as $k(r, t)=\bar{k}\left(r^{2} \psi_{1}(t)+\psi_{2}(t)\right)$ on the rectangle $(0,+\infty) \times(T,+\infty)$.

- $\psi_{1}(t)$ tends to 0 , and $\psi_{2}(t)$ converges to a constant $a \in \mathbb{R}$ as $t$ goes to $+\infty$.

- $\bar{k}$ is of class $C^{1}$, and the derivative $\bar{k}^{\prime}$ does not vanish at a.

Then each level set of $K f(\cdot, t)$ tends to a sphere centered at the centroid of $f$ with respect to the Hausdorff distance as $t$ goes to $+\infty$.

Proof. For each point $x \in \mathbb{R}^{m}$, Lebesgue's dominated convergence theorem implies

$$
\begin{aligned}
\frac{1}{2 \psi_{1}(t)} \operatorname{grad} K f(x, t)= & \int_{\mathbb{R}^{m}} f(y) \bar{k}^{\prime}\left(r^{2} \psi_{1}(t)+\psi_{2}(t)\right)(x-y) d y \\
= & \int_{\mathbb{R}^{m}} f(y) \bar{k}^{\prime}\left(r^{2} \psi_{1}(t)+\psi_{2}(t)\right)\left(x-G_{f}\right) d y \\
& +\int_{\mathbb{R}^{m}} f(y) \bar{k}^{\prime}\left(r^{2} \psi_{1}(t)+\psi_{2}(t)\right)\left(G_{f}-y\right) d y \\
\rightarrow & \left(\bar{k}^{\prime}(a) \int_{\mathbb{R}^{m}} f(y) d y\right)\left(x-G_{f}\right)
\end{aligned}
$$

as $t$ goes to $+\infty$. Hence the limiting position of $\operatorname{grad} K f(x, t)$ is a constant multiple of $x-G_{f}$.

We understand that a set $L$ in $\mathbb{R}^{m}$ is called a time-invariant level set of $K f(\cdot, t)$ if there exists a function $a:(0,+\infty) \rightarrow \mathbb{R}$ which satisfies

$$
K f(x, t)=a(t), x \in L, t>0 .
$$

Corollary 3.14. Let $k$ be as in Theorem 3.13. Moreover, we assume that, for any $x \in \mathbb{R}^{m}$, the potential $K f(x, t)$ converges to $f(x)$ as $t$ tends to $0^{+}$. If any level set of $K f(\cdot, t)$ is time-invariant, then $f$ is rotation invariant about its centroid.

Proof. Fix an arbitrary point $x$ in $\mathbb{R}^{m}$. Thanks to Theorem 3.13 , if $L$ is a level set of $K f(\cdot, t)$ through the point $x$, then $L$ is a sphere centered at the centroid $G_{f}$ with radius $\left|x-G_{f}\right|$. For any point $\xi$ on the sphere $\left|x-G_{f}\right| S^{m-1}+G_{f}$, we have

$$
|f(x)-f(\xi)| \leq|f(x)-K f(x, t)|+|f(\xi)-K f(\xi, t)| \rightarrow 0
$$

as $t$ tends to $0^{+}$, which completes the proof.

Next, we estimate the distance between the centroid $G_{f}$ and the set $\mathcal{K}_{f}(t)$.

Proposition 3.15. Let $f$ be non-zero and non-negative, and $k(r, t)$ strictly decreasing with respect to $r$. Suppose that there exists a non-negative constant $T$ such that if $t>T$, then $k(\cdot, t)$ satisfies the condition $\left(C_{\alpha}^{1}\right)$ for some $\alpha>2$. Moreover, we assume that there exist functions $\psi_{1}, \psi_{2}$ and $\bar{k}$ which satisfy the following conditions:

- $k(r, t)$ can be expressed as $k(r, t)=\bar{k}\left(r^{2} \psi_{1}(t)+\psi_{2}(t)\right)$ on the rectangle $(0, \operatorname{diam}(\operatorname{supp} f)] \times(T,+\infty)$.

- $\bar{k}$ is of class $C^{2}$, the first derivative $\bar{k}^{\prime}$ does not vanish at a constant a $\in$ $\mathcal{I}\left(r^{2}, \psi_{1}, \psi_{2} ; \operatorname{diam}(\operatorname{supp} f), T\right)$, and the second derivative $\bar{k}^{\prime \prime}$ is bounded. 
Then we have

$$
\begin{aligned}
& \frac{\max _{c(t) \in \mathcal{K}_{f}(t)}\left|G_{f}-c(t)\right|}{\operatorname{diam}(\operatorname{supp} f)\left((\operatorname{diam}(\operatorname{supp} f))^{2}\left|\psi_{1}(t)\right|+\left|\psi_{2}(t)-a\right|\right)\left\|\bar{k}^{\prime \prime}\right\|_{\infty} \int_{\mathbb{R}^{m}} f(y) d y} \\
& \inf _{x \in \operatorname{conv}(\operatorname{supp} f)} \int_{\mathbb{R}^{m}} f(y)\left|\bar{k}^{\prime}\left(r^{2} \psi_{1}(t)+\psi_{2}(t)\right)\right| d y
\end{aligned}
$$

for any $t>T$.

Proof. We remark that the integral

$$
\int_{\mathbb{R}^{m}} f(y) \frac{1}{r} \frac{\partial k}{\partial r}(r, t) d y
$$

converges for any $x \in \mathbb{R}^{m}$ if $\alpha>2$ by the same argument as in Lemma 2.4.

Let $t>T$ and fix an arbitrary point $c(t) \in \mathcal{K}_{f}(t)$. We remark that the point $c(t) \in \mathcal{K}_{f}(t)$ satisfies the equation

$c(t) \int_{\mathbb{R}^{m}} f(y) \frac{1}{|c(t)-y|} \frac{\partial k}{\partial r}(|c(t)-y|, t) d y=\int_{\mathbb{R}^{m}} f(y) \frac{y}{|c(t)-y|} \frac{\partial k}{\partial r}(|c(t)-y|, t) d y$.

By the mean value theorem, we have

$$
\begin{aligned}
& \left|c(t)-G_{f}\right| \int_{\mathbb{R}^{m}} f(y) \frac{-1}{|c(t)-y|} \frac{\partial k}{\partial r}(|c(t)-y|, t) d y \\
= & \left|\int_{\mathbb{R}^{m}} f(y) \frac{G_{f}-y}{|c(t)-y|} \frac{\partial k}{\partial r}(|c(t)-y|, t) d y\right| \\
= & 2\left|\psi_{1}(t)\right|\left|\int_{\mathbb{R}^{m}} f(y)\left(G_{f}-y\right)\left(\bar{k}^{\prime}\left(|c(t)-y|^{2} \psi_{1}(t)+\psi_{2}(t)\right)-\bar{k}^{\prime}(a)\right) d y\right| \\
\leq & 2\left|\psi_{1}(t)\right|\left\|\bar{k}^{\prime \prime}\right\|_{\infty}\left|\int_{\mathbb{R}^{m}} f(y)\left(G_{f}-y\right)\left(|c(t)-y|^{2} \psi_{1}(t)+\psi_{2}(t)-a\right) d y\right| \\
\leq & 2\left|\psi_{1}(t)\right|\left\|\bar{k}^{\prime \prime}\right\|_{\infty} \operatorname{diam}(\operatorname{supp} f) \\
& \times\left((\operatorname{diam}(\operatorname{supp} f))^{2}\left|\psi_{1}(t)\right|+\left|\psi_{2}(t)-a\right|\right) \int_{\mathbb{R}^{m}} f(y) d y .
\end{aligned}
$$

On the other hand, we have

$$
\begin{aligned}
& \int_{\mathbb{R}^{m}} f(y) \frac{-1}{|c(t)-y|} \frac{\partial k}{\partial r}(|c(t)-y|, t) d y \\
\geq & 2\left|\psi_{1}(t)\right| \inf _{x \in \operatorname{conv}(\operatorname{supp} f)} \int_{\mathbb{R}^{m}} f(y)\left|\bar{k}^{\prime}\left(r^{2} \psi_{1}(t)+\psi_{2}(t)\right)\right| d y,
\end{aligned}
$$

which completes the proof.

For the end of this subsection, we estimate the difference between the potentials $K f(x, t)$ and $K f^{*}(x, t)$, where $f^{*}$ is the Schwarz symmetrization of $f$. For the reader's convenience, we review the definition of the Schwarz symmetrization.

Let $f$ be non-zero and non-negative. For a real number $b$, we define $(\operatorname{supp} f)_{b}=$ $\left\{x \in \mathbb{R}^{m} \mid f(x) \geq b\right\}$. Let $(\operatorname{supp} f)_{b}^{*}$ be the closed ball centered at the centroid $G_{f}$ with the same mass as $(\operatorname{supp} f)_{b}$. Let

$$
f^{*}(x)=\sup \left\{b \in \mathbb{R} \mid x \in(\operatorname{supp} f)_{b}^{*}\right\} .
$$


Since $\operatorname{supp} f^{*}=(\operatorname{supp} f)_{0}^{*}$, we have

$$
\int_{\mathbb{R}^{m}} f(y) d y=\int_{\mathbb{R}^{m}} f^{*}(y) d y .
$$

Theorem 3.16. Let $f$ be non-zero and non-negative. Suppose that there exist $T \geq 0, \psi_{1}, \psi_{2}$ and $\bar{k}$ which satisfy the following conditions:

- $k(r, t)$ can be expressed as $k(r, t)=\bar{k}\left(r^{2} \psi_{1}(t)+\psi_{2}(t)\right)$ on the rectangle $(0,+\infty) \times(T,+\infty)$.

- $\bar{k}$ is of class $C^{2}$, and $\bar{k}^{\prime \prime}$ is bounded.

For a constant $a \in \mathcal{I}\left(r^{2}, \psi_{1}, \psi_{2} ;+\infty, T\right)$, let

$$
\psi_{\max }(t)=\max \left\{\left|\psi_{1}(t)\right|^{2},\left|\psi_{1}(t)\right|\left|\psi_{2}(t)-a\right|,\left|\psi_{2}(t)-a\right|^{2}\right\} .
$$

Then we have

$$
\begin{aligned}
& \left.\left|K\left(f-f^{*}\right)(x, t)-\bar{k}^{\prime}(a) \psi_{1}(t) \int_{\mathbb{R}^{m}}\right| y\right|^{2}\left(f-f^{*}\right)(y) d y \mid \\
\leq & \psi_{\max }(t)\left\|\bar{k}^{\prime \prime}\right\|_{\infty} \int_{\mathbb{R}^{m}}\left(r^{2}+1\right)^{2}\left|f-f^{*}\right|(y) d y
\end{aligned}
$$

for each $x \in \mathbb{R}^{m}$ and $t>0$.

Proof. Since the centroid of $f^{*}$ coincides with $G_{f}$, the mass equation (3.9) implies

$$
\int_{\mathbb{R}^{m}} y f(y) d y=\int_{\mathbb{R}^{m}} y f^{*}(y) d y .
$$

Hence we obtain

$$
\int_{\mathbb{R}^{m}} x \cdot y\left(f-f^{*}\right)(y) d y=\sum_{j=1}^{m} x_{j} \int_{\mathbb{R}^{m}} y_{j}\left(f-f^{*}\right)(y) d y=0 .
$$

By using the expansion of $\bar{k}$, the mass equation (3.9) and the above formula, we can obtain the conclusion.

Remark 3.17. If $\psi_{2}(t)=a$ for any $t \geq T$, then we can improve the estimation in Theorem 3.16 as

$$
\begin{aligned}
& \left.\left|K\left(f-f^{*}\right)(x, t)-\bar{k}^{\prime}(a) \psi_{1}(t) \int_{\mathbb{R}^{m}}\right| y\right|^{2}\left(f-f^{*}\right)(y) d y \mid \\
\leq & \left|\psi_{1}(t)\right|^{2}\left\|\bar{k}^{\prime \prime}\right\|_{\infty} \int_{\mathbb{R}^{m}} r^{4}\left|f-f^{*}\right|(y) d y .
\end{aligned}
$$

\section{The CASE OF $f=\chi_{\Omega}$}

Let $\Omega$ be a body (the closure of a bounded open set) in $\mathbb{R}^{m}$. In this section, we consider the case where $f$ is the characteristic function $\chi_{\Omega}$. Let

$$
K_{\Omega}(x, t)=K \chi_{\Omega}(x, t)=\int_{\Omega} k(r, t) d y, x \in \mathbb{R}^{m}, t>0, r=|x-y| .
$$

We denote the centroid of $\Omega$ by $G_{\Omega}$ for short. We improve the results on the potential $K f$ in this case. 
4.1. Boundary integral expressions. In this subsection, we assume that $\Omega$ has a piecewise $C^{1}$ boundary. We give boundary integral expressions to the derivatives of $K_{\Omega}(x, t)$ by using Stokes' theorem.

Proposition 4.1. Suppose that $k(\cdot, t)$ satisfies the condition $\left(C_{\alpha}^{1}\right)$ for a positive $t$. Then we have

$$
\frac{\partial K_{\Omega}}{\partial x_{j}}(x, t)=-\int_{\partial \Omega} k(r, t) e_{j} \cdot n(y) d \sigma(y)
$$

for any $x \in \mathbb{R}^{m}$, where $e_{j}$ is the $j$-th unit vector of $\mathbb{R}^{m}$ and $n(y)$ is the unit outer normal of $\partial \Omega$.

Proof. Fix an arbitrary point $x \in \Omega^{c}$. Since $k(r, t)$ is of class $C^{1}$ on $\Omega$ as a function of $y$, we have

$$
\frac{\partial K_{\Omega}}{\partial x_{j}}(x, t)=-\int_{\Omega} \frac{\partial}{\partial y_{j}} k(r, t) d y=-\int_{\partial \Omega} k(r, t) e_{j} \cdot n(y) d \sigma(y)
$$

by Proposition 2.6 and Stokes' theorem.

Fix an arbitrary point $x \in \stackrel{\circ}{\Omega}$ and a small enough $\varepsilon>0$ such that $B_{\varepsilon}(x)$ is contained in $\Omega$. By Proposition 2.6 and the condition $\left(C_{\alpha}^{1}\right)$, we have

$$
\frac{\partial K_{\Omega}}{\partial x_{j}}(x, t)=\lim _{\varepsilon \rightarrow 0} \int_{\Omega \backslash B_{\varepsilon}(x)} \frac{\partial}{\partial x_{j}} k(r, t) d y=-\lim _{\varepsilon \rightarrow 0} \int_{\Omega \backslash B_{\varepsilon}(x)} \frac{\partial}{\partial y_{j}} k(r, t) d y .
$$

Since $k(r, t)$ is of class $C^{1}$ on $\Omega \backslash B_{\varepsilon}(x)$ as a function of $y$, we obtain

$$
\begin{aligned}
\int_{\Omega \backslash B_{\varepsilon}(x)} \frac{\partial}{\partial y_{j}} k(r, t) d y & =\int_{\partial \Omega} k(r, t) e_{j} \cdot n(y) d \sigma(y)-\int_{\partial B_{\varepsilon}(x)} k(r, t) e_{j} \cdot n(y) d \sigma(y) \\
& =\int_{\partial \Omega} k(r, t) e_{j} \cdot n(y) d \sigma(y)
\end{aligned}
$$

by Stokes' theorem. Hence the boundary integral expression holds on $\stackrel{\circ}{\Omega}$.

Since $\alpha-m>-(m-1)=-\operatorname{dim} \partial \Omega$, the boundary integral can be extended to $\partial \Omega$. Hence the first derivative of $K_{\Omega}(\cdot, t)$ is also expressed as such a boundary integral on $\partial \Omega$ by its continuity.

Corollary 4.2. Let $k$ be as in Proposition 4.1. $K_{\Omega}(\cdot, t)$ is of class $C^{2}$ on $\mathbb{R}^{m} \backslash \partial \Omega$, and we have

$$
\frac{\partial^{2} K_{\Omega}}{\partial x_{i} \partial x_{j}}(x, t)=-\int_{\partial \Omega} \frac{\partial}{\partial x_{i}} k(r, t) e_{j} \cdot n(y) d \sigma(y)
$$

for any $x \in \mathbb{R}^{m} \backslash \partial \Omega$.

Proof. Fix an arbitrary $\delta>0$, and let $\Omega_{\delta}=\left\{x \in \Omega \mid \operatorname{dist}\left(x, \Omega^{c}\right)>\delta\right\}$. We show the twice differentiability of $K_{\Omega}(\cdot, t)$ on $\Omega_{\delta}$. For any $x \in \Omega_{\delta}$ and $y \in \partial \Omega$, we have

$$
\left|\frac{\partial}{\partial x_{i}} k(r, t)\right| \leq\left|\frac{\partial k}{\partial r}(r, t)\right| \leq \sup _{x \in \Omega_{\delta}}\left(\sup _{y \in \partial \Omega}\left|\frac{\partial k}{\partial r}(r, t)\right|\right)<+\infty .
$$


By Proposition 4.1 and Lebesgue's dominated convergence theorem, we obtain

$$
\begin{aligned}
& \lim _{\lambda \rightarrow 0} \frac{1}{\lambda}\left(\frac{\partial K_{\Omega}}{\partial x_{j}}\left(x+\lambda e_{i}, t\right)-\frac{\partial K_{\Omega}}{\partial x_{j}}(x, t)\right) \\
= & -\int_{\partial \Omega} \lim _{\lambda \rightarrow 0} \frac{k\left(\left|x+\lambda e_{i}-y\right|, t\right)-k(|x-y|, t)}{\lambda} e_{j} \cdot n(y) d \sigma(y) \\
= & -\int_{\partial \Omega} \frac{\partial}{\partial x_{i}} k(r, t) e_{j} \cdot n(y) d \sigma(y)
\end{aligned}
$$

for any $x \in \Omega_{\delta}$, and it is continuous on $\Omega_{\delta}$.

The same argument above works on $\mathbb{R}^{m} \backslash \Omega$.

Corollary 4.3. Let $k$ be as in Proposition 4.1. If $k(\cdot, t)$ is decreasing and if $\Omega$ is convex, then $K_{\Omega}(\cdot, t)$ is superharmonic on the interior of $\Omega$.

Proof. Since $\Omega$ is convex, we have $(y-x) \cdot n(y)>0$ for any $x \in \stackrel{\circ}{\Omega}$ and $y \in \partial \Omega$. Hence it follows from Corollary 4.2 that

$$
\Delta K_{\Omega}(x, t)=\int_{\partial \Omega} \frac{1}{r} \frac{\partial k}{\partial r}(r, t)(y-x) \cdot n(y) d \sigma(y) \leq 0
$$

for any $x \in \stackrel{\circ}{\Omega}$.

Proposition 4.4. Suppose that $k(\cdot, t)$ satisfies the condition $\left(C_{\alpha}^{2}\right)$ for a positive $t$. Then we have

for any $x \in \mathbb{R}^{m}$.

$$
\frac{\partial^{2} K_{\Omega}}{\partial x_{i} \partial x_{j}}(x, t)=-\int_{\partial \Omega} \frac{\partial}{\partial x_{i}} k(r, t) e_{j} \cdot n(y) d \sigma(y)
$$

Proof. Fix an arbitrary point $x \in \Omega^{c}$. Since $k(r, t)$ is of class $C^{2}$ on $\Omega$ as a function of $y$, we have

$$
\frac{\partial^{2} K_{\Omega}}{\partial x_{i} \partial x_{j}}(x, t)=-\int_{\Omega} \frac{\partial^{2}}{\partial x_{i} \partial y_{j}} k(r, t) d y=-\int_{\partial \Omega} \frac{\partial}{\partial x_{i}} k(r, t) e_{j} \cdot n(y) d \sigma(y)
$$

by Proposition 2.9 and Stokes' theorem.

The rest is similar to the proof of Proposition 4.1 .

Corollary 4.5. Let $k$ be as in Proposition 4.4. $K_{\Omega}(\cdot, t)$ is of class $C^{3}$ on $\mathbb{R}^{m} \backslash \partial \Omega$, and we have

$$
\frac{\partial^{3} K_{\Omega}}{\partial x_{i} \partial x_{j} \partial x_{\ell}}(x, t)=-\int_{\partial \Omega} \frac{\partial^{2}}{\partial x_{i} \partial x_{j}} k(r, t) e_{\ell} \cdot n(y) d \sigma(y)
$$

for any $x \in \mathbb{R}^{m} \backslash \partial \Omega$.

Proof. We can show this statement in the same manner as in Corollary 4.2.

4.2. The location and uniqueness of a $k$-center of $\Omega$. In this subsection, we assume that $k(r, t)$ is strictly decreasing with respect to $r$ for each $t$. We remark that the results in this subsection can be applied to the case where $k(r, t)$ is strictly increasing with respect to $r$ by considering the kernel $-k(r, t)$.

Definition 4.6. A point $x$ is said to be a $k$-center of $\Omega$ at time $t$ if it gives the maximum value of $K_{\Omega}(\cdot, t)$.

In order to improve Proposition 3.2, we introduce the minimal unfolded region from [1]. 
Definition 4.7 ([11). Let $v$ be a point in the unit sphere $S^{m-1}$ and $b \in \mathbb{R}$ a constant. Let $\operatorname{Refl}_{v, b}$ be the reflection of $\mathbb{R}^{m}$ in the hyperplane $\left\{z \in \mathbb{R}^{m} \mid z \cdot v=b\right\}$. Put

$$
\Omega_{v, b}^{+}=\Omega \cap\left\{z \in \mathbb{R}^{m} \mid z \cdot v \geq b\right\}, l(v)=\min \left\{a \in \mathbb{R} \mid \operatorname{Refl}_{v, b}\left(\Omega_{v, b}^{+}\right) \subset \Omega \forall b \geq a\right\} .
$$

Define the minimal unfolded region of $\Omega$ by

$$
U f(\Omega)=\bigcap_{v \in S^{m-1}}\left\{z \in \mathbb{R}^{m} \mid z \cdot v \leq l(v)\right\} .
$$

Remark 4.8 ([1] $)$. $U f(\Omega)$ is compact, convex and contained in conv $\Omega$.

The idea of the proof of the following proposition is due to [2].

Proposition 4.9. For any positive $t$, all the $k$-centers of $\Omega$ at time $t$ belong to $U f(\Omega)$.

Proof. Fix an arbitrary point $x \in(\operatorname{conv} \Omega) \backslash U f(\Omega)$. There exists a direction $v$ such that $x \cdot v>l(v)$. Let $b=(x \cdot v+l(v)) / 2$; then $\operatorname{Refl}_{v, b}\left(\Omega_{v, b}^{+}\right)$is contained in $\Omega$, and $\Omega \backslash\left(\operatorname{Refl}_{v, b}\left(\Omega_{v, b}^{+}\right) \cup \Omega_{v, b}^{+}\right)$has an interior point.

Let $x^{\prime}=\operatorname{Refl}_{v, b}(x)$. By radial symmetry of $k(r, t)$, we have the following equations:

$$
\begin{aligned}
\int_{\operatorname{Refl}_{v, b}\left(\Omega_{v, b}^{+}\right)} k\left(\left|x^{\prime}-y\right|, t\right) d y & =\int_{\Omega_{v, b}^{+}} k(|x-y|, t) d y, \\
\int_{\Omega_{v, b}^{+}} k\left(\left|x^{\prime}-y\right|, t\right) d y & =\int_{\operatorname{Refl}_{v, b}\left(\Omega_{v, b}^{+}\right)} k(|x-y|, t) d y .
\end{aligned}
$$

Since we have $\left|x^{\prime}-y\right|<|x-y|$ for any $y \in \Omega \backslash\left(\operatorname{Refl}_{v, b}\left(\Omega_{v, b}^{+}\right) \cup \Omega_{v, b}^{+}\right)$, the strictly decreasing behavior of $k(\cdot, t)$ implies

$$
K_{\Omega}\left(x^{\prime}, t\right)-K_{\Omega}(x, t)=\int_{\Omega \backslash\left(\operatorname{Refl}_{v, b}\left(\Omega_{v, b}^{+}\right) \cup \Omega_{v, b}^{+}\right)}\left(k\left(\left|x^{\prime}-y\right|, t\right)-k(|x-y|, t)\right) d y>0 ;
$$

that is, $x$ is not a $k$-center.

By the same argument as in [12, we obtain the following theorem.

Theorem 4.10. Let $B$ be a closed $m$-ball in $\mathbb{R}^{m}$. If $\operatorname{Vol}(\Omega)=\operatorname{Vol}(B)$, then

$$
\max _{x \in \mathbb{R}^{m}} K_{\Omega}(x, t) \leq \max _{x \in \mathbb{R}^{m}} K_{B}(x, t),
$$

and equality holds if and only if $\Omega$ is a closed ball.

Proof. Proposition 4.9 guarantees that the center of $B$ coincides with the (unique) $k$-center of $B$ for any $t>0$. By a translation of $\mathbb{R}^{m}$, we may assume that both the center of $B$ and a $k$-center of $\Omega$ at time $t$ coincide with the origin 0 . Since $\left|y^{\prime}\right| \leq\left|y^{\prime \prime}\right|$ for any $y^{\prime} \in B \backslash \Omega$ and $y^{\prime \prime} \in \Omega \backslash B$, we obtain

$$
K_{B}(0, t)-K_{\Omega}(0, t)=\left(\int_{B \backslash \Omega}-\int_{\Omega \backslash B}\right) k(|y|, t) d y \geq 0 .
$$

Equality holds if and only if $\operatorname{Vol}(\Omega \backslash B)=0$; namely, $\Omega$ is a closed ball. 
The uniqueness of a $k$-center of $\Omega$ does not always hold (see Example [5.29). But we can give a sufficient condition of the uniqueness. Moreover, it is very useful for studying the asymptotic behavior of $k$-centers (see Remark 3.10). Therefore, we try to give a new sufficient condition of the uniqueness by using the minimal unfolded region of $\Omega$.

We use the following notation in what follows:

$$
\begin{aligned}
d(\Omega) & =\min \{|z-w| \mid z \in U f(\Omega), w \in \partial \Omega\}, \\
D(\Omega) & =\max \{|z-w| \mid z \in U f(\Omega), w \in \partial \Omega\} .
\end{aligned}
$$

We remark that, in Corollary 3.5. we can replace the assumption " $k(\cdot, t)$ is concave on the interval $(0, \operatorname{diam}(\operatorname{supp} f)]$ " with " $k(\cdot, t)$ is concave on the interval $(0, D(\Omega)]$ " if $f$ is $\chi_{\Omega}$.

Lemma 4.11. Let $\Omega$ be a convex body. If a function $\phi:[0,+\infty) \rightarrow[0,+\infty)$ is concave and strictly increasing on the interval $(d(\Omega), D(\Omega))$, then the radial potential

$$
\Phi_{\Omega}(x)=\int_{S^{m-1}} \phi\left(\rho_{\Omega-x}(v)\right) d \sigma(v), x \in \Omega,
$$

becomes strictly concave on $U f(\Omega)$, where

$$
\rho_{\Omega-x}: S^{m-1} \ni v \mapsto \max \{\lambda \geq 0 \mid x+\lambda v \in \Omega\} \in \mathbb{R}
$$

is the radial function of $\Omega$ with respect to $x \in \Omega$.

The idea of the proof of Lemma 4.11 is due to [10]. For the reader's convenience, we give the proof.

Proof. For any $x$ and $x^{\prime}$ in $U f(\Omega)$, we have

$$
\begin{aligned}
& 2 \Phi_{\Omega}\left(\frac{x+x^{\prime}}{2}\right)-\left(\Phi_{\Omega}(x)+\Phi_{\Omega}\left(x^{\prime}\right)\right) \\
> & \int_{S^{m-1}}\left(\phi\left(\frac{\rho_{\Omega-x}(v)+\rho_{\Omega-x^{\prime}}(v)}{2}\right)-\left(\phi\left(\rho_{\Omega-x}(v)\right)+\phi\left(\rho_{\Omega-x^{\prime}}(v)\right)\right)\right) d \sigma(v) \\
\geq & 0 .
\end{aligned}
$$

Here, the first inequality follows from the convexity of $\Omega$ and the strictly increasing behavior of $\phi$. The second inequality follows from the concavity of $\phi$. Hence we get the conclusion.

Theorem 4.12. Let $\Omega$ be a convex body. If $k$ is positive and if $k(r, t) r^{m-1}$ is decreasing on the interval $(d(\Omega), D(\Omega))$ with respect to $r$, then the potential $K_{\Omega}$ is strictly concave on $U f(\Omega)$.

Proof. Taking the function $\phi$ in Lemma 4.11 as

$$
\phi(\rho)=\int_{0}^{\rho} k(r, t) r^{m-1} d r
$$

we get the conclusion.

Corollary 4.13. Let $\Omega$ and $k$ be as in Theorem 4.12, Then $\Omega$ has a unique $k$ center. 
4.3. Estimation of $K_{\Omega}$ in terms of the Schwarz symmetrization. Let $\Omega^{*}$ be the closed ball centered at the centroid of $\Omega$ with volume $\operatorname{Vol}(\Omega)$. It is easily shown by the definition (3.8) that the Schwarz symmetrization $\left(\chi_{\Omega}\right)^{*}$ coincides with the characteristic function $\chi_{\Omega^{*}}$. In this subsection, we estimate the difference between the potentials $K_{\Omega}(x, t)$ and $K_{\Omega^{*}}(x, t)$ when the kernel $k(r, t)$ is given by the form (3.6). The statements and their proofs in this subsection are based on [4.

Lemma 4.14. Let $k$ be as in Theorem 3.16. Moreover, we assume the following conditions:

- The ratio $\psi_{\max }(t) / \psi_{1}(t)$ tends to zero as $t$ goes to $+\infty$.

- The first derivative $\bar{k}^{\prime}$ does not vanish at a.

Then we have

$$
\lim _{t \rightarrow+\infty} \frac{1}{\bar{k}^{\prime}(a) \psi_{1}(t)}\left(K_{\Omega}-K_{\Omega^{*}}\right)(x, t)=\int_{\mathbb{R}^{m}}|y|^{2}\left(\chi_{\Omega-G_{\Omega}}-\chi_{\Omega^{*}-G_{\Omega}}\right)(y) d y
$$

for each $x \in \mathbb{R}^{m}$, where $\Omega-G_{\Omega}=\left\{y-G_{\Omega} \mid y \in \Omega\right\}$.

Proof. Applying Theorem 3.16 to the case of $f=\chi_{\Omega}$, for each $x \in \mathbb{R}^{m}$, we have

$$
\lim _{t \rightarrow+\infty} \frac{1}{\bar{k}^{\prime}(a) \psi_{1}(t)}\left(K_{\Omega}-K_{\Omega^{*}}\right)(x, t)=\int_{\mathbb{R}^{m}}|y|^{2}\left(\chi_{\Omega}-\chi_{\Omega^{*}}\right)(y) d y .
$$

Since the right hand side is independent of a point $x \in \mathbb{R}^{m}$, we have

$$
\lim _{t \rightarrow+\infty} \frac{1}{\bar{k}^{\prime}(a) \psi_{1}(t)}\left(K_{\Omega}-K_{\Omega^{*}}\right)(x, t)=\lim _{t \rightarrow+\infty} \frac{1}{\bar{k}^{\prime}(a) \psi_{1}(t)}\left(K_{\Omega}-K_{\Omega^{*}}\right)\left(x+G_{\Omega}, t\right) .
$$

By the translation in $\mathbb{R}^{m}$, we have

$$
\left(K_{\Omega}-K_{\Omega^{*}}\right)\left(x+G_{\Omega}, t\right)=\left(K_{\Omega-G_{\Omega}}-K_{\Omega^{*}-G_{\Omega}}\right)(x, t) .
$$

Applying Theorem 3.16 to the case of $f=\chi_{\Omega-G_{\Omega}}$, we obtain the conclusion.

Theorem 4.15. Let $k$ be as in Lemma 4.14, Then we have

$$
\lim _{t \rightarrow+\infty} \frac{1}{\bar{k}^{\prime}(a) \psi_{1}(t)}\left(K_{\Omega}-K_{\Omega^{*}}\right)(x, t) \geq 0
$$

for any $x \in \mathbb{R}^{m}$, and there exists a point $x \in \mathbb{R}^{m}$ which gives equality if and only if $\Omega$ is a closed ball.

Proof. Applying Theorem 4.10 to the case of $k(r, t)=r^{2}$, we have

$$
\int_{\mathbb{R}^{m}}|y|^{2}\left(\chi_{\Omega-G_{\Omega}}-\chi_{\Omega^{*}-G_{\Omega}}\right)(y) d y=\left(\int_{\Omega-G_{\Omega}}-\int_{\Omega^{*}-G_{\Omega}}\right)|y|^{2} d y \geq 0 .
$$

Equality holds if and only if $\Omega$ is a closed ball. Hence Lemma 4.14 implies the conclusion.

Theorem 4.16. Suppose that there exist $T \geq 0, \psi_{1}, \psi_{2}$ and $\bar{k}$ which satisfy the following conditions:

- $k(r, t)$ can be expressed as $k(r, t)=\bar{k}\left(r^{2} \psi_{1}(t)+\psi_{2}(t)\right)$ on the rectangle $(0,+\infty) \times(T,+\infty)$.

- $\psi_{1}(t)$ tends to zero, $\psi_{2}(t)$ converges to a constant $a \in \mathbb{R}$, and the ratio $\left(\psi_{2}(t)-a\right) / \psi_{1}(t)$ converges to a constant as $t$ goes to $+\infty$.

- $\bar{k}$ is of class $C^{1}$, and both $\bar{k}$ and $\bar{k}^{\prime}$ do not vanish at a. 
Let $B \subset \mathbb{R}^{m}$ be a closed $m$-ball. Let

$$
L(x)=\lim _{t \rightarrow+\infty} \frac{1}{\bar{k}^{\prime}(a) \psi_{1}(t)}\left(K_{\Omega}-K_{B}\right)(x, t) .
$$

If there exists an open subset $U \subset \mathbb{R}^{m}$ such that the function $L$ is defined and identically zero on $U$, then $\Omega$ coincides with the ball $B$.

Proof. By the mean value theorem, for each $x \in \mathbb{R}^{m}$ and any large enough $t$, we can choose a constant $\theta=\theta(x, y, t) \in(0,1)$ such that

$$
\begin{aligned}
& \left(K_{\Omega}-K_{B}\right)(x, t)=\bar{k}(a) \int_{\mathbb{R}^{m}}\left(\chi_{\Omega}-\chi_{B}\right)(y) d y \\
& +\int_{\mathbb{R}^{m}} \bar{k}^{\prime}\left(\theta\left(r^{2} \psi_{1}(t)+\psi_{2}(t)\right)+(1-\theta) a\right)\left(r^{2} \psi_{1}(t)+\psi_{2}(t)-a\right)\left(\chi_{\Omega}-\chi_{B}\right)(y) d y .
\end{aligned}
$$

Since $L$ is defined on an open set $U$, we obtain the volume equation $\operatorname{Vol}(\Omega)=$ $\operatorname{Vol}(B)$. Then, for each $x \in U$, we have

$$
L(x)=\int_{\mathbb{R}^{m}}|x-y|^{2}\left(\chi_{\Omega}-\chi_{B}\right)(y) d y .
$$

Since $L$ is identically zero on $U$, we have

$$
0=\operatorname{grad} L(x)=2 \int_{\mathbb{R}^{m}}(x-y)\left(\chi_{\Omega}-\chi_{B}\right)(y) d y=-2 \int_{\mathbb{R}^{m}} y\left(\chi_{\Omega}-\chi_{B}\right)(y) d y .
$$

Therefore, we obtain $G_{\Omega}=G_{B}$; that is, $B=\Omega^{*}$.

In the same manner as in Theorem 4.15, we obtain the conclusion.

Furthermore, we can show the following proposition in the same manner as in Theorem 4.15.

Proposition 4.17. Suppose that there exist $T \geq 0, \psi_{1}$ and $\psi_{2}$ which satisfy the following conditions:

- $k(r, t)$ can be expressed as $k(r, t)=r^{2} \psi_{1}(t)+\psi_{2}(t)$ on $(0,+\infty) \times(T,+\infty)$.

- For any $t>T$, the function $\psi_{1}(t)$ is negative.

Then we have $K_{\Omega}(x, t) \leq K_{\Omega^{*}}(x, t)$ for any $x \in \mathbb{R}^{m}$ and $t>T$. There exist $x \in \mathbb{R}^{m}$ and $t>T$ which give equality if and only if $\Omega$ is a closed ball.

Proof. By the same calculation as in Lemma 4.14 and Theorem 4.15, we have

$$
K_{\Omega}(x, t)-K_{\Omega^{*}}(x, t)=\psi_{1}(t) \int_{\mathbb{R}^{m}}|y|^{2}\left(\chi_{\Omega-G_{\Omega}}-\chi_{\Omega^{*}-G_{\Omega}}\right)(y) d y \leq 0,
$$

where the non-positivity follows from Theorem 4.10, Equality holds if and only if $\Omega$ is a closed ball.

\section{Applications}

5.1. The solution of the Cauchy problem for the heat equation. Let $f$ : $\mathbb{R}^{m} \rightarrow \mathbb{R}$ be a non-zero non-negative bounded measurable function with compact support. In this subsection, we consider a potential of the form

$$
H f(x, t)=\frac{1}{(4 \pi t)^{m / 2}} \int_{\mathbb{R}^{m}} f(y) \exp \left(-\frac{r^{2}}{4 t}\right) d y, x \in \mathbb{R}^{m}, t>0, r=|x-y| .
$$

It is well-known that the potential $H f$ satisfies the Cauchy problem for the heat equation with initial datum $f$. In particular, when $f$ coincides with the characteristic function of a body $\Omega$, we denote the potential $H_{\chi_{\Omega}}$ by $H_{\Omega}(x, t)$ for short. 
Let $\mathcal{H}_{f}(t)$ be the set of points that give the maximum value of the function $H f(\cdot, t): \mathbb{R}^{m} \rightarrow \mathbb{R}$ for each $t>0$; that is,

$$
\mathcal{H}_{f}(t)=\left\{x \in \mathbb{R}^{m} \mid H f(x, t)=\max _{\xi \in \mathbb{R}^{m}} H f(\xi, t)\right\} .
$$

A point in $\mathcal{H}_{f}(t)$ is called a hot spot at time $t$.

In 4], Chavel and Karp studied the asymptotic behavior of a hot spot as $t$ goes to $+\infty$ by computing the derivative of $H f(\cdot, t)$.

Theorem $5.1([4) . \quad$ (1) The set of hot spots is not empty and contained in the convex hull of supp $f$ for any $t>0$.

(2) The set of hot spots converges to the one-point set of the centroid of $f$ with respect to the Hausdorff distance as $t$ goes to $+\infty$.

(These facts also follow from Proposition 3.2 and Corollary 3.9 .)

Proposition 5.2. For each $t>0$, the energy

$$
\int_{\mathbb{R}^{m}} f(x) H f(x, t) d x=\frac{1}{(4 \pi t)^{m / 2}} \int_{\mathbb{R}^{m} \times \mathbb{R}^{m}} f(x) f(y) \exp \left(-\frac{r^{2}}{4 t}\right) d x d y
$$

is maximum in $\mathcal{F}$ (defined in (3.1)) if and only if $f$ is the characteristic function of a closed m-ball up to a zero measure set.

(This fact follows from Theorem 3.6.)

Theorem 5.3 (4]). Each level set of $H f(\cdot, t)$ tends to a sphere centered at the centroid of $f$ with respect to the Hausdorff distance.

(This fact also follows from Theorem 3.13.)

Proposition 5.4 ([4]). We have

$$
\max _{h(t) \in \mathcal{H}_{f}(t)}\left|G_{f}-h(t)\right| \leq \frac{(\operatorname{diam}(\operatorname{supp} f))^{3}}{4 t} \exp \left(-\frac{(\operatorname{diam}(\operatorname{supp} f))^{2}}{4 t}\right)
$$

for any $t>0$.

(This fact also follows from Proposition 3.15)

Theorem 5.5 (4]). We have

$$
\begin{aligned}
& \left|4 t(4 \pi t)^{m / 2} H\left(f-f^{*}\right)(x, t)-\left(-\int_{\mathbb{R}^{m}}|y|^{2}\left(f-f^{*}\right)(y) d y\right)\right| \\
\leq & \frac{1}{4 t} \int_{\mathbb{R}^{m}} r^{4}\left|f-f^{*}\right|(y) d y
\end{aligned}
$$

for any $x \in \mathbb{R}^{m}$ and $t>0$.

(This fact also follows from Remark 3.17)

Proposition 5.6. If $\Omega$ is a convex body with a piecewise $C^{1}$ boundary, then $H_{\Omega}(\cdot, t)$ is superharmonic on the interior of $\Omega$ for any $t>0$.

(This fact follows from Corollary 4.3.)

Proposition 5.7. Let $B$ be a closed $m$-ball in $\mathbb{R}^{m}$. If $\operatorname{Vol}(\Omega)=\operatorname{Vol}(B)$, then we have

$$
\max _{x \in \mathbb{R}^{m}} H_{\Omega}(x, t) \leq \max _{x \in \mathbb{R}^{m}} H_{B}(x, t),
$$

and equality holds if and only if $\Omega$ is a closed ball.

(This fact follows from Theorem 4.10,) 
Theorem $5.8(4])$. For any $x \in \mathbb{R}^{m}$, we have

$$
\lim _{t \rightarrow+\infty} t^{m / 2+1}\left(H_{\Omega}-H_{\Omega^{*}}\right)(x, t) \leq 0,
$$

and equality holds if and only if $\Omega$ is a closed ball.

(This fact also follows from Theorem 4.15)

Theorem 5.9 ([4]). Let $B$ be a closed $m$-ball in $\mathbb{R}^{m}$. If there is an open set $U$ in $\mathbb{R}^{m}$ such that the limit

$$
\lim _{t \rightarrow+\infty} t^{m / 2+1}\left(H_{\Omega}-H_{B}\right)(x, t)
$$

exists and is identically zero on $U$, then $\Omega$ coincides with $B$.

(This fact also follows from Theorem 4.16])

We consider a sufficient condition for the uniqueness of a hot spot. In [6], Jimbo and Sakaguchi indicated that if $t$ is not smaller than $(\operatorname{diam}(\operatorname{supp} f))^{2} / 2$, then the set $\mathcal{H}_{f}(t)$ consists of one point by calculating the Hessian of $H f(\cdot, t)$. We remark that the fact can also be derived from Corollary 3.5. In [8, Magnanini and Sakaguchi indicated that if the initial datum $f$ coincides with the characteristic function of a convex body $\Omega$, then $\Omega$ has a unique hot spot for any $t>0$ by using the logconcavity of $H_{\Omega}(\cdot, t)$ (see also [1]).

We slightly improve the condition of $t$ given in [6] when the initial datum $f$ coincides with $\chi_{\Omega}$ by using the minimal unfolded region. We remark that $\Omega$ is not necessarily convex in our case.

Proposition 5.10. If the initial datum $f$ coincides with the characteristic function of a body $\Omega$, then, for each positive $t$, any hot spot at time $t$ is contained in the minimal unfolded region of $\Omega$.

(This fact follows from Proposition 4.9.)

Lemma 5.11. Let $t>0, p>0$ and $g(s)=\left(s^{2}-2 t\right) \exp \left(-\left(s^{2}+p\right) /(4 t)\right)$.

(1) For any $\delta \in(0,1], g((1-\delta) \sqrt{2 t})+g((1+\delta) \sqrt{2 t})<0$.

(2) $\int_{0}^{\infty} g(s) d s=0$.

Proof. (1) Since $g$ is increasing on the interval $[0, \sqrt{6 t}]$ and decreasing on the interval $[\sqrt{6 t},+\infty)$, it is sufficient to show that the inequality holds for any $0<\delta \leq \sqrt{3}-1$. Let $\phi(\lambda)=(\lambda+1) \exp \left(-\lambda^{2} / 2\right)$ for $2-\sqrt{3} \leq \lambda \leq \sqrt{3}$. It is equivalent to show $\phi(1+\delta)<\phi(1-\delta)$ for any $0<\delta \leq \sqrt{3}-1$. Since $\phi$ is increasing on the interval $[2-\sqrt{3},(-1+\sqrt{5}) / 2]$ and decreasing on the interval $[(-1+\sqrt{5}) / 2, \sqrt{3}]$, it is sufficient to show that the inequality holds for any $(3-\sqrt{5}) / 2 \leq \delta \leq \sqrt{3}-1$. We can easily get $\phi(2-\sqrt{3})>\phi((5-\sqrt{5}) / 2)$, which implies the conclusion.

(2) By integration by parts, we have

$$
\int_{0}^{\infty} s^{2} \exp \left(-\frac{s^{2}}{4 t}\right) d s=\int_{0}^{\infty} s \frac{\partial}{\partial s}\left(-2 t \exp \left(-\frac{s^{2}}{4 t}\right)\right) d s=2 t \int_{0}^{\infty} \exp \left(-\frac{s^{2}}{4 t}\right) d s,
$$

which completes the proof.

Definition 5.12. Let us use the notation in Definition 4.7 A function $\gamma: S^{m-1} \rightarrow$ $\mathbb{R}$ is called a folding function of a body $\Omega$ if, for each $v \in S^{m-1}$ and any $b \geq \gamma(v)$, $\operatorname{Refl}_{v, b}\left(\Omega_{v, b}^{+}\right)$is contained in $\Omega$. We denote the set of continuous folding functions of 
$\Omega$ by $C F(\Omega)$. Let $W_{\gamma}(\Omega)$ be the Wulff shape associated with a function $\gamma \in C F(\Omega)$; that is, $W_{\gamma}(\Omega)$ is defined as

$$
W_{\gamma}(\Omega)=\bigcap_{v \in S^{m-1}}\left\{x \in \mathbb{R}^{m} \mid x \cdot v \leq \gamma(v)\right\} .
$$

Define the minimal unfolded Wulff shape of $\Omega$ by

$$
W(\Omega)=\bigcap_{\gamma \in C F(\Omega)} W_{\gamma}(\Omega)
$$

Remark 5.13. We remark that $U f(\Omega)$ is contained in $W(\Omega)$. Furthermore, in general, $W(\Omega)$ is not contained in $\Omega$ if $\Omega$ is not convex, while it is always contained in the convex hull of $\Omega$.

We use the following notation in what follows:

$$
\tilde{D}(\Omega)=\max \{|z-w| \mid z \in U f(\Omega), w \in W(\Omega)\} .
$$

Theorem 5.14. If the initial datum $f$ coincides with the characteristic function of a body $\Omega$ and if $t \geq \tilde{D}(\Omega)^{2} / 2$, then $\Omega$ has a unique hot spot at time $t$.

Proof. Let us use the notation in Definition 4.7 and Lemma 5.11 in what follows.

Fix a point $x$ in $U f(\Omega)$ and a time $t \geq \tilde{D}(\Omega)^{2} / 2$. Let $\tau=x_{1}+\sqrt{2 t}$. Direct computation shows

$$
\frac{\partial^{2} H_{\Omega}}{\partial x_{1}^{2}}(x, t)=\frac{1}{4 t^{2}(4 \pi t)^{m / 2}} \int_{\Omega}\left(\left(x_{1}-y_{1}\right)^{2}-2 t\right) \exp \left(-\frac{r^{2}}{4 t}\right) d y .
$$

Therefore, if both $\Omega_{e_{1}, \tau}$ and $\Omega_{-e_{1}, \tau}$ are empty, then we have $\left(\partial^{2} H_{\Omega} / \partial x_{1}^{2}\right)(x, t)<0$ (see Figure 1).

We consider the case where either $\Omega_{e_{1}, \tau}$ or $\Omega_{-e_{1}, \tau}$ is not empty. Then we have $\operatorname{Refl}_{e_{1}, \tau}\left(\Omega_{e_{1}, \tau}^{+}\right) \subset \Omega$. Put

$$
L_{1}(y)=\left\{z=y+t e_{1} \mid t \in \mathbb{R}\right\}, L_{1}\left(y ; z_{1} \geq c\right)=L_{1}(y) \cap\left\{z \in \mathbb{R}^{m} \mid z_{1} \geq c\right\} .
$$

We apply Lemma 5.11 to the case of $p=\sum_{\ell=2}^{m}\left(x_{\ell}-y_{\ell}\right)^{2}$. By Fubini's theorem, we have

$$
\begin{aligned}
\frac{\partial^{2} H_{\Omega}}{\partial x_{1}^{2}}(x, t) & =\frac{1}{(4 \pi t)^{m / 2} 4 t^{2}} \int_{\Omega} g\left(x_{1}-y_{1}\right) d y \\
& =\frac{1}{(4 \pi t)^{m / 2} 4 t^{2}} \int_{\Omega \mid e_{1}^{\perp}}\left(\int_{\Omega \cap L_{1}\left(\left(x_{1}, \bar{y}\right)\right)} g\left(x_{1}-y_{1}\right) d y_{1}\right) d \bar{y}
\end{aligned}
$$

where $\Omega \mid e_{1}^{\perp}$ denotes the image of the orthogonal projection from $\Omega$ to $e_{1}^{\perp}$, and $\bar{y}=\left(y_{2}, \ldots, y_{m}\right)$. We remark that $g\left(x_{1}-y_{1}\right)$ is obviously negative for any $y$ in the band $\left\{z \in \mathbb{R}^{m} \mid x_{1}-\tau \leq z_{1} \leq x_{1}+\tau\right\}$.

If $\operatorname{Refl}_{e_{1}, \tau}\left(\Omega \cap L_{1}\left(\left(x_{1}, \bar{y}\right) ; z_{1} \geq \tau\right)\right)$ is contained in $L_{1}\left(\left(x_{1}, \bar{y}\right) ; z_{1} \geq x_{1}\right)$, then the first assertion of Lemma 5.11 implies

$$
\int_{\operatorname{Refl}_{e_{1}, \tau}\left(\Omega \cap L_{1}\left(\left(x_{1}, \bar{y}\right) ; z_{1} \geq \tau\right)\right) \cup\left(\Omega \cap L_{1}\left(\left(x_{1}, \bar{y}\right) ; z_{1} \geq \tau\right)\right)} g\left(x_{1}-y_{1}\right) d y_{1}<0 .
$$

Otherwise, the second assertion of Lemma 5.11 implies

$$
\int_{\Omega \cap L_{1}\left(\left(x_{1}, \bar{y}\right) ; z_{1} \geq x_{1}\right)} g\left(x_{1}-y_{1}\right) d y_{1}<\int_{L_{1}\left(\left(x_{1}, \bar{y}\right) ; z_{1} \geq x_{1}\right)} g\left(x_{1}-y_{1}\right) d y_{1}=0 .
$$


These arguments also work for the other side $\left\{z \in \mathbb{R}^{m} \mid z_{1} \leq x_{1}\right\}$. Hence we get $\left(\partial^{2} H_{\Omega} / \partial x_{1}^{2}\right)(x, t)<0$ for any $x \in U f(\Omega)$ (see Figure 2).

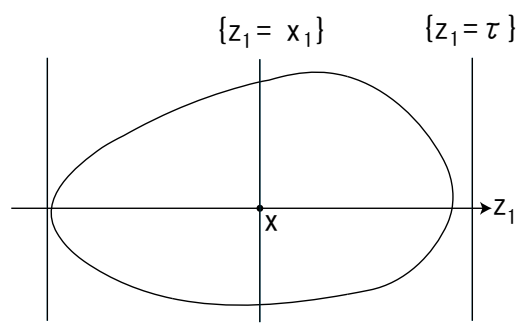

Figure 1. The case of $\Omega_{ \pm e_{1}, \tau}=\emptyset$.

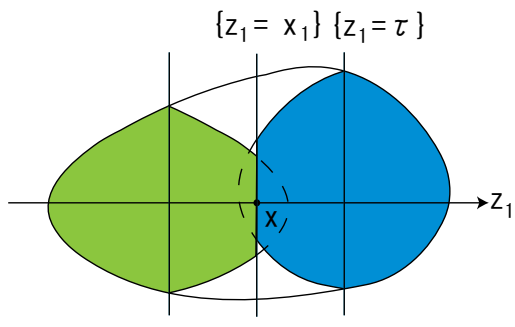

Figure 2. The case of $\Omega_{ \pm e_{1}, \tau} \neq \emptyset$.

By radial symmetry of the heat kernel, we obtain the conclusion.

Remark 5.15. Theorem 5.14 does not provide a real improvement of the result of [6] in the case of $f=\chi_{\Omega}$ since the relation $\tilde{D}(\Omega)<\operatorname{diam} \Omega$ does not always hold.

5.2. The Poisson integral. Let $f: \mathbb{R}^{m} \rightarrow \mathbb{R}$ be a non-zero non-negative bounded measurable function with compact support. Let

$$
\operatorname{Pf}(x, t)=\frac{2 t}{\sigma_{m}\left(S^{m}\right)} \int_{\mathbb{R}^{m}} \frac{f(y)}{\left(r^{2}+t^{2}\right)^{(m+1) / 2}} d y, x \in \mathbb{R}^{m}, t>0, r=|x-y|,
$$

which is called the Poisson integral. It is well-known that $P f$ satisfies the Laplace equation for the upper half space $\left\{(x, t) \mid x \in \mathbb{R}^{m}, t>0\right\}$ with boundary datum $f$. In this subsection, we consider the asymptotic behavior of maximum points of $\operatorname{Pf}(\cdot, t): \mathbb{R}^{m} \rightarrow \mathbb{R}$ as $t$ goes to $+\infty$.

Proposition 5.16. The set of maximum points of $P f(\cdot, t)$ is not empty and contained in the convex hull of supp $f$ for any $t>0$.

(This fact follows from Proposition 3.2.)

We denote the set of maximum points of $\operatorname{Pf}(\cdot, t)$ by $\mathcal{P}_{f}(t)$; that is,

$$
\mathcal{P}_{f}(t)=\left\{x \in \mathbb{R}^{m} \mid \operatorname{Pf}(x, t)=\max _{\xi \in \mathbb{R}^{m}} \operatorname{Pf}(\xi, t)\right\} .
$$

Proposition 5.17. If $t \geq \sqrt{m+2} \operatorname{diam}(\operatorname{supp} f)$, then $\mathcal{P}_{f}(t)$ becomes a one-point set.

(This fact follows from Corollary [3.5.)

Proposition 5.18. For each $t>0$, the energy

$$
\int_{\mathbb{R}^{m}} f(x) \operatorname{Pf}(x, t) d x=\frac{2 t}{\sigma_{m}\left(S^{m}\right)} \int_{\mathbb{R}^{m} \times \mathbb{R}^{m}} \frac{f(x) f(y)}{\left(r^{2}+t^{2}\right)^{(m+1) / 2}} d x d y
$$

is maximum in $\mathcal{F}$ (defined in (3.1)) if and only if $f$ is the characteristic function of a closed $m$-ball up to a zero measure set.

(This fact follows from Theorem 3.6.)

Proposition 5.19. The set $\mathcal{P}_{f}(t)$ converges to the one-point set of the centroid of $f$ with respect to the Hausdorff distance as $t$ goes to $+\infty$. 
Proof. The Poisson integral $P f(x, t)$ corresponds to the case where $\varphi(r)=r^{2}$, $\psi_{1}(t)=t^{-2}, \psi_{2}(t)=0$ and $\bar{k}(s)=(1+s)^{-(m+1) / 2}$ in Corollary 3.9 .

Proposition 5.20. Each level set of the Poisson integral P $f(\cdot, t)$ tends to a sphere centered at the centroid of $f$ with respect to the Hausdorff distance.

(This fact follows from Theorem 3.13.)

Proposition 5.21. We have

$$
\max _{p(t) \in \mathcal{P}_{f}(t)}\left|G_{f}-p(t)\right| \leq \frac{(m+3)(\operatorname{diam}(\operatorname{supp} f))^{3}}{2 t^{2}}\left(\left(\frac{\operatorname{diam}(\operatorname{supp} f)}{t}\right)^{2}+1\right)^{(m+3) / 2}
$$

for any $t>0$.

(This fact follows from Proposition 3.15)

Proposition 5.22. We have

$$
\begin{aligned}
& \left|\frac{\sigma_{m}\left(S^{m}\right) t^{m+2}}{m+1} P\left(f-f^{*}\right)(x, t)-\left(-\int_{\mathbb{R}^{m}}|y|^{2}\left(f-f^{*}\right)(y) d y\right)\right| \\
\leq & \frac{m+3}{2 t^{2}} \int_{\mathbb{R}^{m}} r^{4}\left|f-f^{*}\right|(y) d y
\end{aligned}
$$

for any $x \in \mathbb{R}^{m}$ and $t>0$.

(This fact follows from Remark 3.17.)

Let $\Omega$ be a body in $\mathbb{R}^{m}$. Let us consider the case of $f=\sigma_{m}\left(S^{m}\right) \chi_{\Omega} / 2$ in what follows. Let

$$
A_{\Omega}(x, h)=\frac{\sigma_{m}\left(S^{m}\right)}{2} P \chi_{\Omega}(x, h)=\int_{\Omega} \frac{h}{\left(r^{2}+h^{2}\right)^{(m+1) / 2}} d y .
$$

In this case, the Poisson integral $A_{\Omega}(x, h)$ has a geometric meaning as below: Let $x$ be a point in $\mathbb{R}^{m}$ and $h$ a positive real number. Define the map $p_{(x, h)}: \Omega \rightarrow S^{m}$ by

$$
p_{(x, h)}(y)=\frac{(y, 0)-(x, h)}{|(y, 0)-(x, h)|}=\frac{(y-x, 0-h)}{\sqrt{r^{2}+h^{2}}} .
$$

The solid angle of $\Omega$ at a point $(x, h)$ is given by the spherical $m$-volume of the image of $p_{(x, h)}$. By direct calculation, we can show that the solid angle coincides with the Poisson integral $A_{\Omega}(x, h)$. We remark that the function $A_{\Omega}(x, h)$ coincides with Shibata's total brightness of a triangular park when $\Omega$ is a triangle in $\mathbb{R}^{2}$.

Definition 5.23. A point $x$ in $\mathbb{R}^{m}$ is called a spatial illuminating center of $\Omega$ of height $h$ if it gives the maximum value of $A_{\Omega}(\cdot, h): \mathbb{R}^{m} \rightarrow \mathbb{R}$.

Proposition 5.24. If $\Omega$ is a convex body with a piecewise $C^{1}$ boundary, then $A_{\Omega}(\cdot, h)$ is superharmonic on the interior of $\Omega$ for any $h>0$.

(This fact follows from Corollary 4.3.)

Proposition 5.25. For any positive $h$, every spatial illuminating center of $\Omega$ of height $h$ belongs to the minimal unfolded region of $\Omega$.

(This fact follows from Proposition 4.9.) 
Proposition 5.26. Let $B$ be a closed $m$-ball in $\mathbb{R}^{m}$. If $\operatorname{Vol}(\Omega)=\operatorname{Vol}(B)$, then we have

$$
\max _{x \in \mathbb{R}^{m}} A_{\Omega}(x, h) \leq \max _{x \in \mathbb{R}^{m}} A_{B}(x, h),
$$

and equality holds if and only if $\Omega$ is a closed ball.

(This fact follows from Theorem 4.10.)

Proposition 5.27. For any $x \in \mathbb{R}^{m}$, we have

$$
\lim _{h \rightarrow+\infty} h^{m+2}\left(A_{\Omega}-A_{\Omega^{*}}\right)(x, h) \leq 0,
$$

and equality holds if and only if $\Omega$ is a closed ball.

(This fact follows from Theorem 4.15.)

Proposition 5.28. Let $B$ be a closed $m$-ball in $\mathbb{R}^{m}$. If there exists an open set $U$ in $\mathbb{R}^{m}$ such that the limit

$$
\lim _{h \rightarrow+\infty} h^{m+2}\left(A_{\Omega}-A_{B}\right)(x, h)
$$

exists and is identically zero on $U$, then $\Omega$ coincides with $B$.

(This fact follows from Theorem 4.16.)

We also study the uniqueness of a spatial illuminating center of $\Omega$ of height $h$. We remark that the uniqueness does not always hold. Let us see it in an example when $m=1$.

Example 5.29. Let $\Omega$ be a disjoint union of two intervals with the same length: $\Omega=[-R,-1] \cup[1, R]$, where $R>1$. By direct computation, we obtain the following results (see Figure [3):

(1) If $0<h<\sqrt{R+(R+1) \sqrt{R}}$, then

$$
x_{ \pm}(h)= \pm \sqrt{\sqrt{R\left((R+1)^{2}+4 h^{2}\right)}-\left(R+h^{2}\right)}
$$

are the spatial illuminating centers of $\Omega$

(2) If $h \geq \sqrt{R+(R+1) \sqrt{R}}$, then the origin is the unique spatial illuminating center of $\Omega$.

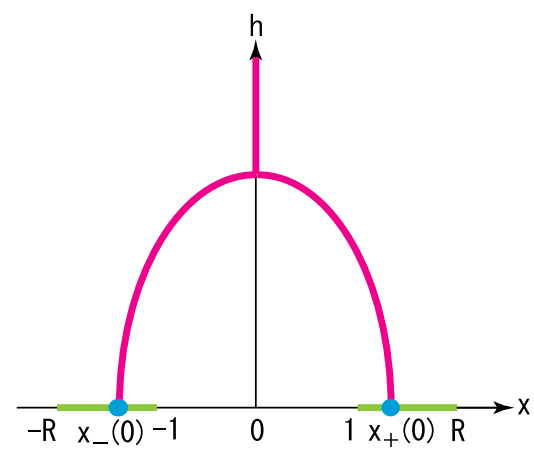

FiguRE 3. The locus of spatial illuminating centers of height $h>0$. 
Proposition 5.30. If $h \geq \sqrt{m+2} D(\Omega)$, then $\Omega$ has a unique spatial illuminating center of height $h$, where $D(\Omega)$ is defined in (4.2).

(This fact follows from the remark before Lemma 4.11,)

Let us improve Proposition 5.30 in the same manner as in Theorem 5.14 ,

Lemma 5.31. Let $p>0$ and $g(s)=\left((m+2) s^{2}-p\right)\left(s^{2}+p\right)^{-(m+5) / 2}$.

(1) We have $g((1-\delta) \sqrt{p /(m+2)})+g((1+\delta) \sqrt{p /(m+2)})<0$ for any $\delta \in$ $(0,1]$.

(2) $\int_{0}^{+\infty} g(s) d s=0$.

Proof. (1) It is sufficient to show that the inequality holds for any $\delta \in(0, \sqrt{3}-1]$ since $g$ is increasing on the interval $[0, \sqrt{3 p /(m+2)}]$ and decreasing on the interval $[\sqrt{3 p /(m+2)},+\infty)$. It is equivalent to show $\phi_{m}(1+\delta)<\phi_{m}(1-\delta)$ for any $\delta \in(0, \sqrt{3}-1]$, where $\phi_{m}(\lambda)=(\lambda+1)\left(\lambda^{2}+m+2\right)^{-(m+5) / 2}$. Let $\lambda_{c}(m)$ be the unique positive critical point of $\phi_{m}$. Then we have $\phi_{m}(1+\delta)<\phi_{m}(1-\delta)$ for any $\delta \in\left(0,1-\lambda_{c}(m)\right]$. Since $\lambda_{c}(m)$ is strictly increasing and tends to $(-1+\sqrt{5}) / 2$ as $m$ goes to $+\infty$, it is sufficient to show $\phi_{m}(2-\sqrt{3})>\phi_{m}((5-\sqrt{5}) / 2)$. The sequence $\left\{\phi_{m}(2-\sqrt{3}) / \phi_{m}((5-\sqrt{5}) / 2)\right\}$ is decreasing, and we can show

$$
\begin{aligned}
& \lim _{m \rightarrow+\infty} \frac{\phi_{m}(2-\sqrt{3})}{\phi_{m}((5-\sqrt{5}) / 2)} \\
= & \frac{(2-\sqrt{3})+1}{(5-\sqrt{5}) / 2+1} \exp \left(\frac{1}{2}\left(\left(\frac{5-\sqrt{5}}{2}\right)^{2}-(2-\sqrt{3})^{2}\right)\right) \\
> & 1,
\end{aligned}
$$

which completes the proof.

(2) By changing the variable $s \mapsto \sqrt{p} \tan \theta$, we can obtain the conclusion.

Theorem 5.32. If $h \geq \sqrt{m+2} \tilde{D}(\Omega)$, then $\Omega$ has a unique spatial illuminating center of height $h$, where $\tilde{D}(\Omega)$ is defined in (5.3).

Proof. Let us use the notation in Definition 4.7 and Lemma 5.31 in what follows.

Fix a point $x$ in $U f(\Omega)$ and a height $h \geq \sqrt{m+2} \tilde{D}(\Omega)$. Let $\zeta=x_{1}+h / \sqrt{m+2}$. Then the half-space $\left\{z \in \mathbb{R}^{m} \mid z_{1} \geq \zeta\right\}$ contains the right side of the hyperboloid in Figure 4 (and 5). If both $\Omega_{e_{1}, \zeta}$ and $\Omega_{-e_{1}, \zeta}$ are empty, then we have the negativity of $\left(\partial^{2} A_{\Omega} / \partial x_{1}^{2}\right)(x, h)$ in the same manner as in Theorem 5.14 (see Figure 4).

We consider the case where either $\Omega_{e_{1}, \zeta}$ or $\Omega_{-e_{1}, \zeta}$ is not empty. Then we have $\operatorname{Refl}_{e_{1}, \zeta}\left(\Omega_{e_{1}, \zeta}^{+}\right) \subset \Omega$. We apply Lemma 5.31 to the case of $p=\sum_{\ell=2}^{m}\left(x_{\ell}-y_{\ell}\right)^{2}+h^{2}$. By Fubini's theorem, we have

$$
\begin{aligned}
\frac{\partial^{2} A_{\Omega}}{\partial x_{1}^{2}}(x, h) & =(m+1) h \int_{\Omega} g\left(x_{1}-y_{1}\right) d y \\
& =(m+1) h \int_{\Omega \mid e_{1}^{\perp}}\left(\int_{\Omega \cap L_{1}\left(\left(x_{1}, \bar{y}\right)\right)} g\left(x_{1}-y_{1}\right) d y_{1}\right) d \bar{y},
\end{aligned}
$$

where $L_{1}(y)$ is defined in (5.4). We remark that the integral of $g\left(x_{1}-y_{1}\right)$ over the inside of the hyperboloid $\left\{z \in \mathbb{R}^{m} \mid(m+2)\left(x_{1}-z_{1}\right)^{2}-\sum_{\ell=2}^{m}\left(x_{\ell}-z_{\ell}\right)^{2}-h^{2} \leq 0\right\}$ 
is obviously negative. By Lemma 5.31 and the same argument as in Theorem 5.14 , we can show $\left(\partial^{2} A_{\Omega} / \partial x_{1}^{2}\right)(x, h)<0$ (see Figure 5).

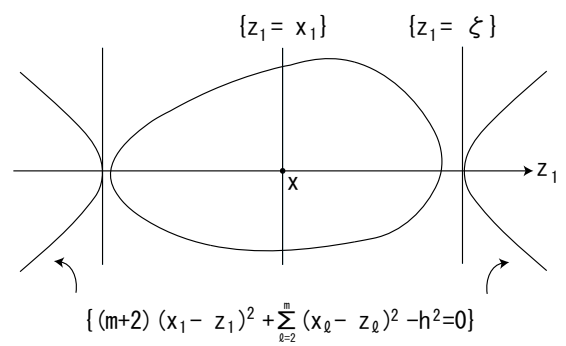

Figure 4. The case of $\Omega_{ \pm e_{1}, \zeta}=\emptyset$.

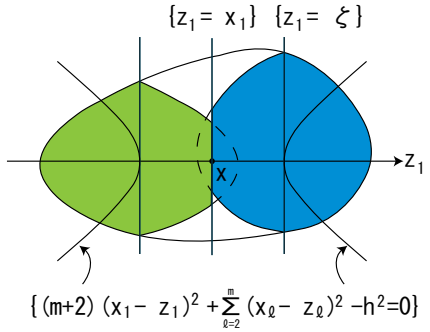

Figure 5. The case of $\Omega_{ \pm e_{1}, \zeta} \neq \emptyset$.

In Example 5.29, we showed that, for any body $\Omega$, the uniqueness of a spatial illuminating center of height $h$ does not always hold without the condition for $h$. Next we study the conditions of the uniqueness for $\Omega$.

Proposition 5.33. Let $\Omega$ be a convex body such that $U f(\Omega)$ is contained in the interior of $\Omega$. If $h \leq \sqrt{2 /(m-1)} d(\Omega)$, then $\Omega$ has a unique spatial illuminating center of height $h$, where $d(\Omega)$ is defined in (4.2).

(This fact follows from Corollary 4.13 .)

Corollary 5.34. Let $\Omega$ be as in Proposition 5.33. If $\sqrt{(m+2)(m-1) / 2} \tilde{D}(\Omega) \leq$ $d(\Omega)$, then $\Omega$ has a unique spatial illuminating center for any $h$.

(This fact follows from Theorem 5.32 and Proposition 5.33 .)

Lemma 5.35. Let us use the notation in Definition 5.12 in what follows. Let $\tilde{\Omega}$ be a compact convex set. For any positive $\delta, W\left(\tilde{\Omega}+\delta B^{m}\right)$ is contained in $\tilde{\Omega}$, where $B^{m}$ is the closed unit $m$-ball and $\tilde{\Omega}+\delta B^{m}$ is the $\delta$-parallel body $\left\{z+\delta w \mid z \in \tilde{\Omega}, w \in B^{m}\right\}$.

Proof. Fix an arbitrary direction $v \in S^{m-1}$, and let $\gamma(v)=\max _{y \in \tilde{\Omega}}(y \cdot v)$. We remark that $\tilde{\Omega}$ is contained in the half-space $\left\{z \in \mathbb{R}^{m} \mid z \cdot v \leq \gamma(v)\right\}$. It is sufficient to show that $\gamma(v)$ is a folding function of $\tilde{\Omega}+\delta B^{m}$.

Since $\tilde{\Omega}+\delta B^{m}$ is expressed as $\tilde{\Omega}+\delta B^{m}=\bigcup_{y \in \tilde{\Omega}} B_{\delta}(y)$, we obtain that, for any $b \geq \gamma(v)$,

$$
\begin{aligned}
\operatorname{Refl}_{v, b}\left(\left(\tilde{\Omega}+\delta B^{m}\right)_{v, b}^{+}\right) & =\operatorname{Refl}_{v, b}\left(\bigcup_{y \in \tilde{\Omega}} B_{\delta}(y) \cap\left\{z \in \mathbb{R}^{m} \mid z \cdot v \geq b\right\}\right) \\
& =\bigcup_{y \in \tilde{\Omega}} \operatorname{Refl}_{v, b}\left(B_{\delta}(y) \cap\left\{z \in \mathbb{R}^{m} \mid z \cdot v \geq b\right\}\right) \\
& \subset \tilde{\Omega}+\delta B^{m},
\end{aligned}
$$

which completes the proof.

Theorem 5.36. If $\Omega$ can be expressed as $\Omega=\tilde{\Omega}+\delta B^{m}$ with a compact convex set $\tilde{\Omega}$ and a constant $\delta \geq \sqrt{(m+2)(m-1) / 2} \operatorname{diam} \tilde{\Omega}$, then $\Omega$ has a unique spatial illuminating center for any $h$. 
Proof. Remark 5.13 and Lemma 5.35 imply that $U f(\Omega) \subset W(\Omega) \subset \tilde{\Omega}$. Therefore, we obtain

$$
\sqrt{\frac{(m+2)(m-1)}{2}} \tilde{D}(\Omega) \leq \sqrt{\frac{(m+2)(m-1)}{2}} \operatorname{diam} \tilde{\Omega} \leq \delta \leq d(\Omega)
$$

if $\delta \geq \sqrt{(m+2)(m-1) / 2}$ diam $\tilde{\Omega}$. Hence Corollary 5.34]implies the conclusion.

5.3. $r^{\alpha-m}$-potentials. In this subsection, we introduce the $r^{\alpha-m}$-potential of a body $\Omega$ for $\alpha>0$ from [11] and consider its generalization. Define the function $V_{\Omega}^{(\alpha)}: \mathbb{R}^{m} \rightarrow \mathbb{R}$ for $\alpha>0$ by

$$
V_{\Omega}^{(\alpha)}(x)=\left\{\begin{array}{ll}
\operatorname{sign}(m-\alpha) \int_{\Omega} r^{\alpha-m} d y & (0<\alpha \neq m), \\
-\int_{\Omega} \log r d y & (\alpha=m),
\end{array} \quad x \in \mathbb{R}^{m}, r=|x-y| .\right.
$$

Definition 5.37 (11). A point $x$ in $\mathbb{R}^{m}$ is called an $r^{\alpha-m}$-center of $\Omega$ if it gives the maximum value of $V_{\Omega}^{(\alpha)}$.

Theorem 5.38 ([1]). (1) For any positive $\alpha, r^{\alpha-m}$-centers of $\Omega$ exist and belong to the minimal unfolded region of $\Omega$.

(2) If $\Omega$ is convex and $0<\alpha \leq 1$, then $\Omega$ has a unique $r^{\alpha-m}$-center.

(3) If $\alpha \geq m+1$, then $\Omega$ has a unique $r^{\alpha-m}$-center.

(These facts also follow from Propositions 3.2 and 4.9 and Corollaries 4.13 and 3.5.)

Proposition 5.39. Let $B$ be a closed $m$-ball in $\mathbb{R}^{m}$. If $\operatorname{Vol}(\Omega)=\operatorname{Vol}(B)$, then

$$
\max _{x \in \mathbb{R}^{m}} V_{\Omega}^{(\alpha)}(x) \leq \max _{x \in \mathbb{R}^{m}} V_{B}^{(\alpha)}(x),
$$

and equality holds if and only if $\Omega$ is a closed ball.

(This fact follows from Theorem 4.10.)

Proposition 5.40. We have $V_{\Omega}^{(m+2)}(x) \leq V_{\Omega^{*}}^{(m+2)}(x)$ for any $x \in \mathbb{R}^{m}$. There exists a point $x \in \mathbb{R}^{m}$ which gives equality if and only if $\Omega$ is a closed ball.

(This fact follows from Proposition 4.17)

In [11, O'Hara showed that, as $\alpha$ goes to $+\infty$, an $r^{\alpha-m}$-center converges to the point that gives the minimum value of the function $\mathbb{R}^{m} \ni x \mapsto \max _{y \in \Omega}|x-y| \in \mathbb{R}$. The same argument also works for the potential

$$
V^{(\alpha)} f(x)= \begin{cases}\operatorname{sign}(m-\alpha) \int_{\mathbb{R}^{m}} f(y) r^{\alpha-m} d y & (0<\alpha \neq m), \\ -\int_{\mathbb{R}^{m}} f(y) \log r d y & (\alpha=m),\end{cases}
$$

where $f: \mathbb{R}^{m} \rightarrow \mathbb{R}$ is a non-zero non-negative bounded measurable function with compact support.

Proposition 5.41. There exists a maximizer of $V^{(\alpha)} f$, and all of those are contained in the convex hull of the support of $f$.

(These facts follow from Proposition 3.2.) 
Definition 5.42. (1) A point $x$ in $\mathbb{R}^{m}$ is called an $r^{\alpha-m}$-center of $f$ if it gives the maximum value of $V^{(\alpha)} f$.

(2) A point is called an $r^{\infty}$-center or a min-max point of $f$ if it gives the minimum value of the function $\mathbb{R}^{m} \ni x \mapsto \max _{y \in \operatorname{supp} f}|x-y| \in \mathbb{R}$.

Proposition 5.43. If $\alpha \geq m+1$, then $f$ has a unique $r^{\alpha-m}$-center.

(This fact follows from Corollary 3.5.)

Proposition 5.44. For each $0<\alpha \neq m$, the energy of $V^{(\alpha)} f$

$$
\int_{\mathbb{R}^{m}} f(x) V^{(\alpha)} f(x) d x=\operatorname{sign}(m-\alpha) \int_{\mathbb{R}^{m} \times \mathbb{R}^{m}} f(x) f(y) r^{\alpha-m} d x d y
$$

is maximum in $\mathcal{F}$ (defined in (3.1)) if and only if $f$ is the characteristic function of a closed $m$-ball up to a zero measure set.

(This fact follows from Theorem 3.6.)

For the readers' convenience, we give the proof of the theorem that an $r^{\alpha-m_{-}}$ center of $f$ converges to the min-max point of $f$ as $\alpha$ goes to $+\infty$ with slight modification of the argument in [1].

Lemma 5.45. If $f$ is continuous, then we have

$$
\lim _{\alpha \rightarrow+\infty}\left(-V^{(\alpha)} f(x)\right)^{1 / \alpha}=\operatorname{esssup}_{y \in \operatorname{supp} f}|x-y|=\max _{y \in \operatorname{supp} f}|x-y| .
$$

Proof. Since $f$ is bounded on $\mathbb{R}^{m}$, for any $x \in \mathbb{R}^{m}$, we have

$$
\begin{aligned}
\left(-V^{(\alpha)} f(x)\right)^{1 / \alpha} & \leq\|f\|_{\infty}^{1 / \alpha}\left(\int_{\operatorname{supp} f}|x-y|^{\alpha-m} d y\right)^{1 / \alpha} \\
& \rightarrow \operatorname{esssup}_{y \in \operatorname{supp} f}|x-y| \\
& =\max _{y \in \operatorname{supp} f}|x-y|
\end{aligned}
$$

as $\alpha$ goes to $+\infty$.

We show the opposite inequality. Fix a point $x \in \mathbb{R}^{m}$ and an arbitrary $\varepsilon>0$. There exists a point $y^{\prime} \in \operatorname{supp} f$ which gives a positive value to $f$ and satisfies

$$
\left|x-y^{\prime}\right| \geq \max _{y \in \operatorname{supp} f}|x-y|-\varepsilon .
$$

Since $f$ is continuous on $\mathbb{R}^{m}$, there exists a positive constant $\delta$ such that, for any $y \in B_{\delta}\left(y^{\prime}\right)$, we have $f(y) \geq f\left(y^{\prime}\right) / 2$. Hence we obtain

$$
\begin{aligned}
\left(-V^{(\alpha)} f(x)\right)^{1 / \alpha} & \geq\left(\frac{f\left(y^{\prime}\right)}{2}\right)^{1 / \alpha}\left(\int_{B_{\delta}\left(y^{\prime}\right)}|x-y|^{\alpha-m} d y\right)^{1 / \alpha} \\
& \rightarrow \operatorname{esssup}_{y \in B_{\delta}\left(y^{\prime}\right)}|x-y| \\
& \geq \max _{y \in \operatorname{supp} f}|x-y|-\varepsilon,
\end{aligned}
$$

which completes the proof.

Theorem 5.46. Suppose that $f$ is continuous. If the body $\operatorname{supp} f$ satisfies the Poincaré condition inside (that is, for any point $y$ on $\partial(\operatorname{supp} f)$, there exist positive constants $\varepsilon$ and $\theta$ such that $B_{\varepsilon}(y) \cap \operatorname{supp} f$ contains a cone of revolution with vertex $y$ and cone angle $\theta)$, then an $r^{\alpha-m}$-center converges to the min-max point of $f$ as $\alpha$ goes to $+\infty$. 
Proof. Let $c(\alpha)$ be the (unique) $r^{\alpha-m}$-center for $\alpha \geq m+1$ and $c_{\infty}$ the min-max point of $f$. Define the function $\rho: \mathbb{R}^{m} \ni x \mapsto \max _{y \in \operatorname{supp} f}|x-y|$.

We show that, for any $\varepsilon>0$, there exists an $\alpha_{\varepsilon} \geq m+1$ such that if $\alpha>$ $\alpha_{\varepsilon}$, then $c(\alpha)$ is contained in $B_{\varepsilon}\left(c_{\infty}\right)$. Suppose that there is no such $\alpha_{\varepsilon}$. Since the family of $r^{\alpha-m}$-centers $\{c(\alpha)\}$ is contained in $\operatorname{conv}(\operatorname{supp} f)$, we can choose a strictly increasing sequence $\left\{\alpha_{\ell}\right\}$ such that $\alpha_{\ell}$ diverges to $+\infty$ and the sequence $\left\{c\left(\alpha_{\ell}\right)\right\}$ converges to a point $c \in \operatorname{conv}(\operatorname{supp} f)$ as $\ell$ goes to $+\infty$. Since $c \neq c_{\infty}$, we have $\rho(c)>\rho\left(c_{\infty}\right)$. Let $\delta=\left(\rho(c)-\rho\left(c_{\infty}\right)\right) / 2>0$. There exists a point $y^{\prime} \in \partial(\operatorname{conv}(\operatorname{supp} f))$ such that $\rho(c)=\left|c-y^{\prime}\right|$. Since $f$ is continuous on $\mathbb{R}^{m}$, for any $x \in B_{\delta}(c)$, we have

$$
-V^{(\alpha)} f(x)>(\rho(c)-2 \delta)^{\alpha-m} \int_{B_{\delta}\left(y^{\prime}\right)} f(y) d y=\rho\left(c_{\infty}\right)^{\alpha-m} \int_{B_{\delta}\left(y^{\prime}\right)} f(y) d y .
$$

On the other hand, we have

$$
-V^{(\alpha)} f\left(c_{\infty}\right)<\|f\|_{\infty} \int_{B_{\rho\left(c_{\infty}\right)}\left(c_{\infty}\right)}\left|c_{\infty}-y\right|^{\alpha-m} d y=\frac{1}{\alpha}\|f\|_{\infty} \sigma\left(S^{m-1}\right) \rho\left(c_{\infty}\right)^{\alpha} .
$$

Since there exists a large enough $\alpha \geq m+1$ such that

$$
\alpha>\rho\left(c_{\infty}\right)^{m}\|f\|_{\infty} \sigma\left(S^{m-1}\right) / \int_{B_{\delta}\left(y^{\prime}\right)} f(y) d y,
$$

we have $V^{(\alpha)} f(x)<V^{(\alpha)} f\left(c_{\infty}\right)$ for any $x \in B_{\delta}(c)$, which contradicts the maximality of $V^{(\alpha)} f(c(\alpha))$.

\section{NON-EUCLIDEAN CASES}

In this section, we consider the same problems on the unit sphere $S^{m}$ or on the Lorenz model of the hyperbolic space $\mathbb{H}^{m}$. We can obtain the same results as in the Euclidean case by parallel arguments.

Let $S^{m}$ be the unit $m$-sphere in $\mathbb{R}^{m+1}$ and $\sigma_{m}$ the spherical Lebesgue measure of $S^{m}$. Let $f: S^{m} \rightarrow \mathbb{R}$ be a bounded measurable function. Consider a kernel $k:(0, \pi] \times(0,+\infty) \rightarrow \mathbb{R}$ such that $k(\cdot, t):(0, \pi] \rightarrow \mathbb{R}$ satisfies any of the conditions $(B),\left(C_{\alpha}^{0}\right),\left(C_{\alpha}^{1}\right)$ or $\left(C_{\alpha}^{2}\right)$ for each $t>0$. Let

$$
K f(x, t)=\int_{S^{m}} f(y) k(r, t) d \sigma_{m}(y), x \in S^{m}, t>0,
$$

where $r=\operatorname{dist}_{S^{m}}(x, y)=\arccos x \cdot y$.

Throughout this section, we use the polar coordinate

$$
\begin{aligned}
& \left(\cos \theta_{1}, \sin \theta_{1} \cos \theta_{2}, \ldots, \sin \theta_{1} \cdots \sin \theta_{m-1} \cos \theta_{m}, \sin \theta_{1} \cdots \sin \theta_{m-1} \sin \theta_{m}\right), \\
& \left(\theta_{1}, \ldots, \theta_{m-1}, \theta_{m}\right) \in[0, \pi] \times \cdots \times[0, \pi] \times[0,2 \pi],
\end{aligned}
$$

for a point $x=x\left(\theta_{1}, \ldots, \theta_{m}\right)$ in $S^{m}$.

By the same arguments as in Lemma 3.1 and Propositions 2.3, 2.6 and 2.9, we obtain the following proposition.

Proposition 6.1. (1) Under the condition $(B), K f(\cdot, t)$ is defined and semicontinuous on $S^{m}$.

(2) Under the condition $\left(C_{\alpha}^{0}\right), K f(\cdot, t)$ is defined and continuous on $S^{m}$. 
(3) Under the condition $\left(C_{\alpha}^{1}\right), K f(\cdot, t)$ is of class $C^{1}$ on $S^{m}$, and we have

$$
\frac{\partial}{\partial \theta_{j}} K f(x, t)=\int_{S^{m}} f(y) \frac{\partial}{\partial \theta_{j}} k(r, t) d \sigma_{m}(y)
$$

for any $x \in S^{m}$.

(4) Under the condition $\left(C_{\alpha}^{2}\right), K f(\cdot, t)$ is of class $C^{2}$ on $S^{m}$, and we have

$$
\frac{\partial^{2}}{\partial \theta_{i} \partial \theta_{j}} K f(x, t)=\int_{S^{m}} f(y) \frac{\partial^{2}}{\partial \theta_{i} \partial \theta_{j}} k(r, t) d \sigma_{m}(y)
$$

for any $x \in S^{m}$.

We assume that $f$ is non-zero and non-negative in what follows. Moreover, we assume that $k(r, t)$ is strictly decreasing with respect to $r$ for each $t$. We remark that the results in this section can be applied to the case where $k(r, t)$ is strictly increasing with respect to $r$ by considering the kernel $-k(r, t)$.

We understand that a set $X$ in $S^{m}$ is said to be spherical convex if the cone $\bigcup_{x \in X}\left\{\xi \in \mathbb{R}^{m+1} \mid \xi=t x \exists t \in[0,1]\right\}$ is convex in $\mathbb{R}^{m+1}$. We define the spherical convex hull of $X$ by the minimal spherical convex set which contains $X$. We denote the spherical convex hull of $X$ by the same symbol conv $X$ as in the Euclidean case. We remark that if $X$ is not contained in a hemisphere, then the spherical convex hull of $X$ coincides with $S^{m}$.

By the same argument as in Proposition 3.2, we obtain the following proposition.

Proposition 6.2. For any $t>0$, there exists a maximum point of $K f(\cdot, t)$, and all of those are contained in the spherical convex hull of supp $f$.

Definition 6.3. A point $x \in S^{m}$ is called a spherical $k$-center of $f$ at time $t$ if it gives the maximum value of $K f(\cdot, t)$.

For a set $X$ in $S^{m}$, we let

$$
X^{*}=\bigcap_{x \in X}\left\{\xi \in S^{m} \mid \xi \cdot x \leq 0\right\},
$$

which is called the polar set of $X$. We remark that a set $X$ in $S^{m}$ is contained in a hemisphere if and only if the polar set of $X$ is not empty.

Theorem 6.4. Suppose that $k(\cdot, t)$ satisfies the spherical condition $\left(C_{\alpha}^{2}\right)$ for a positive $t$. Moreover, we assume that $\operatorname{conv}(\operatorname{supp} f)$ is contained in $-(\operatorname{supp} f)^{*}$. If $k(\cdot, t)$ is concave on $(0, \operatorname{diam}(\operatorname{supp} f)]$, then $f$ has a unique spherical $k$-center at time $t$.

Proof. By radial symmetry of the kernel $k(r, t)$, it suffices to show that the second derivative $\left(\partial^{2} / \partial \theta_{1}^{2}\right) k(r, t)$ is non-positive for any $R \in S O(m+1), x \in R(\operatorname{conv}(\operatorname{supp} f))$ and $y \in R(\operatorname{supp} f)$. Since $\operatorname{conv}(\operatorname{supp} f)$ is contained in $-(\operatorname{supp} f)^{*}$, we have

$$
\begin{aligned}
\frac{\partial^{2}}{\partial \theta_{1}^{2}} k(r, t)= & \frac{\left(\partial^{2} k / \partial r^{2}\right)(r, t)}{1-(x \cdot y)^{2}}\left(\frac{\partial x}{\partial \theta_{1}} \cdot y\right)^{2} \\
& +\frac{(\partial k / \partial r)(r, t)(x \cdot y)}{\left(1-(x \cdot y)^{2}\right)^{3 / 2}}\left(1-\left((x \cdot y)^{2}+\left(\frac{\partial x}{\partial \theta_{1}} \cdot y\right)^{2}\right)\right) \\
\leq & 0
\end{aligned}
$$

for any $R \in S O(m+1), x \in R(\operatorname{conv}(\operatorname{supp} f))$ and $y \in R(\operatorname{supp} f)$.

By the same argument as in Theorem 3.8, we can show the following theorem. 
Theorem 6.5. Let $k$ be as in the spherical version of Lemma 3.7. Moreover, we assume that $\operatorname{conv}(\operatorname{supp} f)$ is contained in $-(\operatorname{supp} f)^{*}$. For any strictly increasing sequence $\left\{t_{\ell}\right\}$ and any spherical $k$-center $c\left(t_{\ell}\right) \in \mathcal{K}_{f}\left(t_{\ell}\right)$, if $\left\{t_{\ell}\right\}$ diverges to $+\infty$ as $\ell$ goes to $+\infty$, then the distance between $c\left(t_{\ell}\right)$ and $\mathcal{C}(\varphi, f)$ converges to 0 as $\ell$ goes to $+\infty$.

Let

$$
V^{(\alpha)} f(x)= \begin{cases}\operatorname{sign}(m-\alpha) \int_{S^{m}} f(y) r^{\alpha-m} d \sigma_{m}(y) & (0<\alpha \neq m), \\ -\int_{S^{m}} f(y) \log r d \sigma_{m}(y) & (\alpha=m) .\end{cases}
$$

By Theorem 6.4 we can show that if $\alpha \geq m+1$, and if $\operatorname{conv}(\operatorname{supp} f)$ is contained in $-(\operatorname{supp} f)^{*}$, then $V^{(\alpha)} f$ has a unique maximizer. By the same argument as in Theorem 5.46, we can show the following theorem.

Theorem 6.6. Suppose that $f$ satisfies the same assumptions as in the spherical version of Theorem 5.46. If $\operatorname{conv}(\operatorname{supp} f)$ is contained in $-(\operatorname{supp} f)^{*}$, then a spherical $r^{\alpha-m}$-center of $f$ converges to the spherical min-max point of $f$ as $\alpha$ goes to $+\infty$.

Let $\Omega$ be a body in $S^{m}$. Define the potential

$$
K_{\Omega}(x, t)=K \chi_{\Omega}(x, t)=\int_{\Omega} k(r, t) d \sigma_{m}(y), x \in S^{m}, t>0 .
$$

If $\Omega$ is contained in a hemisphere, we can define the spherical minimal unfolded region of $\Omega$ in the same manner as in Definition 4.7. Hence we can show the following theorem by the same argument as in Theorem 4.10

Theorem 6.7. Let $\Omega$ be a body in $S^{m}$ and $B$ a closed spherical $m$-ball. If $\sigma_{m}(\Omega)=$ $\sigma_{m}(B)$, and if $\Omega$ is contained in a hemisphere, then we have

$$
\max _{x \in S^{m}} K_{\Omega}(x, t) \leq \max _{x \in S^{m}} K_{B}(x, t),
$$

and equality holds if and only if $\Omega$ is a closed spherical ball.

Let $\langle\cdot, \cdot\rangle$ denote the indefinite inner product of $\mathbb{R}^{m+1}$ given by $\langle x, y\rangle=-x_{0} y_{0}+$ $x_{1} y_{1}+\cdots+x_{m} y_{m}, \mathbb{R}_{1}^{m+1}$ the $(m+1)$-dimensional Euclidean space with $\langle\cdot, \cdot\rangle$, and $\mathbb{H}^{m}$ the Lorents model of the hyperbolic space, $\mathbb{H}^{m}=\left\{x \in \mathbb{R}_{1}^{m+1} \mid\langle x, x\rangle=-1, x_{0}>0\right\}$.

Since the inner product $\langle x, y\rangle$ is always negative for any points $x$ and $y$ in $\mathbb{H}^{m}$, we obtain the same results as in the spherical case without the assumption "conv $(\operatorname{supp} f)$ is contained in $-(\operatorname{supp} f)^{*}$ ".

\section{ApPEndix}

Let $\Omega$ be a body in $S^{m}$. In this section, we always assume that $\Omega^{*}$ is not empty; that is, $\Omega$ is contained in a hemisphere. Furthermore, we assume that $\Omega$ satisfies the Poincaré condition inside; namely, for any point $y$ on the boundary of $\Omega$, there exist positive constants $\varepsilon$ and $\theta$ such that $B_{\varepsilon}(y) \cap \Omega$ contains a spherical cone of revolution with vertex $y$ and cone angle $\theta$.

For a point $x \in-\stackrel{\circ}{\Omega^{*}}$, we let $p_{x}$ be the projection from $\Omega$ to the tangent plane ( $m$-space) of $S^{m}$ at $x$ and $A_{\Omega}$ the map that assigns the area (spherical $m$-volume) 
of $p_{x}(\Omega)$ to a point $x$ in $-\stackrel{\circ}{\Omega^{*}}$ :

$$
p_{x}: \Omega \ni y \mapsto \frac{y}{x \cdot y} \in T_{x} S^{m}, A_{\Omega}(x)=\operatorname{Area}\left(p_{x}(\Omega)\right) .
$$

Since the Jacobian of $p_{x}(y)$ is given by $J p_{x}(y)=(x \cdot y)^{-(m+1)}$, we have

$$
A_{\Omega}(x)=\int_{\Omega} \frac{1}{(x \cdot y)^{m+1}} d \sigma_{m}(y) .
$$

In 12 , the author showed that there is a unique $A_{\Omega}$-minimizer. We generalize the notion in the same manner as in this paper. Let $\tilde{k}:(0,1] \rightarrow \mathbb{R}$ be a strictly monotonic function which satisfies the following condition $\left(\tilde{C}_{\alpha}^{2}\right)$ :

$\left(\tilde{C}_{\alpha}^{2}\right) \alpha \leq 0, \tilde{k}$ is of class $C^{2}$ on the interval $(0,1]$, and $\tilde{k}(\lambda)=O\left(\lambda^{\alpha-m}\right)$ as $\lambda \rightarrow 0^{+}$.

Let

$$
\tilde{K}_{\Omega}(x)=\int_{\Omega} \tilde{k}(x \cdot y) d \sigma_{m}(y), x \in-\stackrel{\circ}{\Omega}^{*} .
$$

We assume that $\tilde{k}$ is decreasing in what follows. We remark that the results in this section can be applied to the case where $\tilde{k}$ is increasing by considering the kernel $-\tilde{k}$.

Theorem 7.1. If $\tilde{k}$ is convex on the interval $(0,1]$, then there exists a unique $\tilde{K}_{\Omega}$-minimizer.

Proof. Since $\tilde{K}_{\Omega}$ is continuous on $-\stackrel{\circ}{\Omega}^{*}$ and diverges to $+\infty$ as $x$ approaches the boundary of $-\Omega^{*}, \tilde{K}_{\Omega}$ has a minimizer.

We show the uniqueness of a minimizer of $\tilde{K}_{\Omega}$. By radial symmetry the kernel of $\tilde{K}_{\Omega}$, it is sufficient to show that the second derivative $\left(\partial^{2} / \partial \theta_{1}^{2}\right) \tilde{K}_{\Omega}(x)$ is positive for any $x \in-\stackrel{\circ}{\Omega}^{*}$, where we used the coordinate (6.2). By direct computation, we have

$$
\frac{\partial^{2}}{\partial \theta_{1}^{2}} \tilde{K}_{\Omega}(x)=\int_{\Omega}\left(\tilde{k}^{\prime \prime}(x \cdot y)\left(\frac{\partial x}{\partial \theta_{1}} \cdot y\right)^{2}-\tilde{k}^{\prime}(x \cdot y) x \cdot y\right) d \sigma_{m}(y)>0
$$

for any $x \in-\stackrel{\circ}{\Omega^{*}}$, which completes the proof.

By the same argument as in Theorem 4.10, we can show the following theorem.

Theorem 7.2. Let $B$ be a closed spherical $m$-ball. If $\sigma_{m}(\Omega)=\sigma_{m}(B)$, then

$$
\min _{x \in-B^{*}} \tilde{K}_{B}(x) \leq \min _{x \in-\Omega^{*}} \tilde{K}_{\Omega}(x),
$$

and equality holds if and only if $\Omega$ is a closed spherical ball.

\section{ACKNOWLEDGEMENTS}

The author would like to express his deep gratitude to Professor Jun O'Hara (his advisor), Professor Kazuhiro Kurata and Professor Rolando Magnanini. Professor O'Hara suggested this topic of study and gave kind advice during the writing of this paper. Professor Kurata informed the author of the papers [4,6], which are related on hot spots. Professor Magnanini gave valuable advice on geometry of solutions of PDE's. The author also thanks the referee for a careful reading and the very kind report. 


\section{REFERENCES}

[1] Herm Jan Brascamp and Elliott H. Lieb, On extensions of the Brunn-Minkowski and Prékopa-Leindler theorems, including inequalities for log concave functions, and with an application to the diffusion equation, J. Functional Analysis 22 (1976), no. 4, 366-389. MR0450480 (56 \#8774)

[2] Lorenzo Brasco and Rolando Magnanini, The heart of a convex body, Geometric properties for parabolic and elliptic PDE's, Springer INdAM Ser., vol. 2, Springer, Milan, 2013, pp. 49-66, DOI 10.1007/978-88-470-2841-8_4. MR.3050226

[3] Lorenzo Brasco, Rolando Magnanini, and Paolo Salani, The location of the hot spot in a grounded convex conductor, Indiana Univ. Math. J. 60 (2011), no. 2, 633-659, DOI 10.1512/iumj.2011.60.4578. MR.2963787

[4] Isaac Chavel and Leon Karp, Movement of hot spots in Riemannian manifolds, J. Analyse Math. 55 (1990), 271-286, DOI 10.1007/BF02789205. MR1094719 (92b:58223)

[5] B. Gidas, Wei Ming Ni, and L. Nirenberg, Symmetry and related properties via the maximum principle, Comm. Math. Phys. 68 (1979), no. 3, 209-243. MR544879 (80h:35043)

[6] Shuichi Jimbo and Shigeru Sakaguchi, Movement of hot spots over unbounded domains in $\mathbf{R}^{N}$, J. Math. Anal. Appl. 182 (1994), no. 3, 810-835, DOI 10.1006/jmaa.1994.1123. MR.1272155(95b:35081)

[7] Lester L. Helms, Potential theory, Universitext, Springer-Verlag London, Ltd., London, 2009. MR2526019 (2011a:31001)

[8] Rolando Magnanini and Shigeru Sakaguchi, On heat conductors with a stationary hot spot, Ann. Mat. Pura Appl. (4) 183 (2004), no. 1, 1-23, DOI 10.1007/s10231-003-0077-1. MR2044330(2005b:35104)

[9] Frank Morgan, A round ball uniquely minimizes gravitational potential energy, Proc. Amer. Math. Soc. 133 (2005), no. 9, 2733-2735 (electronic), DOI 10.1090/S0002-9939-05-08070-6. MR2146221(2006a:76117)

[10] Maria Moszyńska, Looking for selectors of star bodies, Special issue dedicated to Helmut R. Salzmann on the occasion of his 70th birthday, Geom. Dedicata 83 (2000), no. 1-3, 131-147, DOI 10.1023/A:1005208712952. MR:1800016 (2001k:52010)

[11] Jun O'Hara, Renormalization of potentials and generalized centers, Adv. in Appl. Math. 48 (2012), no. 2, 365-392, DOI 10.1016/j.aam.2011.09.003. MR2873883

[12] Shigehiro Sakata, Extremal problems for the central projection, J. Geom. 103 (2012), no. 1, 125-129, DOI 10.1007/s00022-012-0113-7. MR2944554

[13] James Serrin, A symmetry problem in potential theory, Arch. Rational Mech. Anal. 43 (1971), 304-318. MR0333220 (48 \#11545)

[14] Katsuyuki Shibata, Where should a streetlight be placed in a triangle-shaped park? Elementary integro-differential geometric optics, http://www1.rsp.fukuoka-u.ac.jp/kototoi/ shibataaleph-sjs.pdf

Global Education Center, Waseda University, 1-104 Totsuka-machi, Shinjuku-ku,

TOKYO 169-8050, JAPAN

E-mail address: s.sakata@aoni.waseda.jp

URL: http://tmubdell.math.se.tmu.ac.jp/sakata/ 